\title{
Physiological mechanism of enhancing salinity tolerance of Gleditsia sinensis Lam. by arbuscular mycorrhizal fungi
}

Jinping Wang

nanjing forestry university

\section{Bo Zhang}

university of california, davis

Jinchi Zhang ( $\square$ zhangjc8811@gmail.com )

Nanjing Forestry University https://orcid.org/0000-0002-0517-7214

G. Geoff Wang

Clemson University

Jie Lin

Nanjing Forestry University

Xin Liu

nanjing forestry university

Cuiyu Liu

nanjing forestry university

Shilin Ma

Nanjing forestry University

\section{Research article}

Keywords: arbuscular mycorrhizal fungi, Gleditsia sinensis Lam., salinity tolerance, growth parameters, physiological mechanism

Posted Date: December 12th, 2019

DOl: https://doi.org/10.21203/rs.2.18799/v1

License: (c) (i) This work is licensed under a Creative Commons Attribution 4.0 International License. Read Full License 
1 Up-regulation of autophagy by low concentration of salicylic acid delays methyl jasmonate-induced leaf senescence

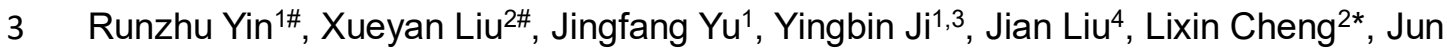

4 Zhou $^{1 *}$

51 MOE Key Laboratory of Laser Life Science \& Guangdong Provincial Key Laboratory of

6 Laser Life Science, College of Biophotonics, South China Normal University, Guangzhou

$7 \quad 510631$, China

82 Department of Critical Care Medicine, Shenzhen People's Hospital, The Second Clinical

$9 \quad$ Medicine College of Jinan University, Shenzhen 518020, China

103 Luoyang Tmaxtree Biotechnology Co., Ltd, Luoyang 471023, China

114 Fujian Provincial Key Laboratory of Plant Functional Biology, College of Life Sciences,

12 Fujian Agriculture and Forestry University, Fuzhou 350002, China

$13{ }^{*}$ Correspondence authors: Jun Zhou, zhoujun@scnu.edu.cn; Lixin Cheng,

14 easonlcheng@gmail.com;

15 \# These authors contributed equally to this work.

Running title: Role of autophagy in LCSA-delayed leaf senescence. 


\section{ABSTRACT}

Crosstalk between salicylic acid (SA) and jasmonic acid (JA) signaling plays an important role in molecular regulation of plant senescence. Our previous works found that SA could delay methyl jasmonate (MeJA)-induced leaf senescence in a concentration-dependent manner. Here, the effect of low concentration of SA (LCSA) application on MeJA-induced leaf senescence was further assessed. High-throughput sequencing (RNA-Seq) results showed that LCSA did not have dominant effects on the genetic regulatory pathways of basal metabolism like nitrogen metabolism, photosynthesis and glycolysis. The ClusterONE was applied to identify discrete gene modules based on protein-protein interaction (PPI) network. Interestingly, an autophagy-related (ATG) module was identified in the differentially expressed genes (DEGs) that exclusively induced by MeJA together with LCSA. RT-qPCR confirmed that the expression of most of the determined ATG genes were upregulated by LCSA. Remarkably, in contrast to wild type (Col-0), LCSA cannot alleviate the leaf yellowing phenotype in autophagy defective mutants (atg5-1 and atg7-2) upon MeJA treatment. Confocal results showed that LCSA increased the number of autophagic bodies accumulated in the vacuole during MeJA-induced leaf senescence. Collectively, our work revealed up-regulation of autophagy by LCSA as a key regulator to alleviate MeJA-induced leaf senescence.

Key Words: autophagy; gene modules; jasmonic acid; leaf senescence; RNA-Seq; salicylic acid 


\section{INTRODUCTION}

Senescence in green plants is a complex and orderly regulated process that is crucial for transiting from nutrient assimilation to nutrient remobilization (Masclaux et al., 2000; Quirino et al., 2000; Lim et al., 2003; Yoshida, 2003; Schippers, 2015). During senescence, the most visible characteristic is leaf yellowing, which is the consequence of a succession of changes in cellular physiology including chlorophyll degradation and photosynthetic activity reduction (Lim et al., 2003; Yoshida, 2003). Chloroplast as an early senescence signaling response organelle, its dismantling plays an important role in the major nitrogen source recycling and remobilization (Avila-Ospina et al., 2014). The progression of leaf senescence can be prematurely induced by multiple environmental and endogenous factors, such as temperature, light, humidity and phytohormones (Lim et al., 2007). Hormone signaling pathways play roles at all the stages of leaf senescence, including the initiation, progression, and the terminal phases of senescence (Lim et al., 2007). Recent progresses show that senescence can be coordinately regulated by several phytohormones like cytokinins, ethylene, abscisic acid, salicylic acid (SA), and jasmonic acid (JA) (Gan and Amasino, 1995; van der Graaff et al., 2006; Hung and Kao, 2004; He et al., 2002; Morris et al., 2000). However, the detailed molecular mechanisms for these phytohormone signals in plant senescence remain poorly understood.

JA has been known as a key plant hormone for promoting senescence, based on the findings that exogenously applied methyl jasmonate (MeJA, methyl ester of JA) leads to a rapid loss of chlorophyll content and accompany with reduction of photochemical efficiency (Yue et al., 2012; Ji et al., 2016). Studies with JA-insensitive mutant coronatine insensitive 1 (coi1) that exhibited defective senescence response to MeJA treatment (He et al., 2002), supporting the notion that JA signaling pathway is crucial for leaf senescence. Some other evidences indicate that SA is also involved in plant senescence (Morris et al., 2000; Chai et al., 2014). The concentration of endogenous SA increases to upregulate several senescence-associated genes during leaf senescence (Morris et al., 2000; Yoshimoto et al., 2009). However, such genetic regulatory mechanisms are abolished in plants defective in the SA signaling or biosynthetic pathway (npr1 and pad4 mutants, and NahG transgenic plants) (Morris et al., 2000). Crosstalk between MeJA and SA has been broadly documented in plant defense response, which commonly manifests as a reciprocal antagonism pattern (Thaler et al., 2012). Evidence suggests that antagonistic interactions between SA and MeJA modulate the expression of a senescence-specific transcription factor WRKY53, showing induced by SA, but repressed by MeJA (Miao and Zentgraf, 
2007). Overall, mechanisms determining the specificity and coordination between SA and JA still need to be further explored.

Most of phytohormones have both stimulatory and inhibitory effects on the growth and metabolism of higher plants in a dose dependent manner. It seems that SA functions in the same way on the physiological and biochemical processes of plants ( $\mathrm{Ji}$ et al., 2016; Pasternak et al., 2019). Low-concentration SA (hereafter as LCSA) at below 50 micromole $(\mu \mathrm{M})$ promotes adventitious roots and altered architecture of the root apical meristem, whereas high-concentration SA (greater than $50 \mu \mathrm{M}$ ) inhibits root growth (Pasternak et al., 2019). Interestingly, we previously demonstrated that MeJA-induced leaf senescence could be delayed by LCSA (1-50 $\mu \mathrm{M})$, but accelerated when the concentration higher than 100 MM (Ji et al., 2016). Our other related works have verified such high dose of SA greatly activates NPR1 (nonexpressor of pathogenesis-related genes 1) translocation into nucleus, thereby promoting leaf senescence (Chai et al., 2014). Based on the dose dependent effect of SA, Pasternak et al. (2019) proposes that at low levels it acts as a developmental regulator and at high levels it acts as a stress hormone.

Autophagy is associated with plant senescence as defective mutants display early and strong yellowing leaf symptoms (Hanaoka et al., 2002; Xiong et al., 2005; Avila-Ospina et al., 2014; Li et al., 2014). Autophagy negatively regulates cell death by controlling NPR1dependent SA signaling during senescence in Arabidopsis (Yoshimoto et al., 2009). The senescence process always accompanies with the equilibrium between oxidative and antioxidative capacities of the plant, which creates a characteristic oxidative environment resulting in the production of reactive oxygen species (ROS) and more toxic derivatives (Bhattacharjee, 2005). Moreover, autophagy is involved in the degradation of oxidized proteins under oxidative stress conditions in Arabidopsis (Xiong et al., 2007). Actually, there is a complicated interplay between ROS and autophagy, i.e., ROS can induce autophagy while autophagy be able to reduce ROS production (Signorelli et al., 2009). Our previous studies showed that LCSA application delays senescence by enhancing the activities of antioxidant enzymes and restricting reactive oxygen species (ROS) accumulation in MeJAtreated leaves (Ji et al., 2016). However, it is still unclear whether autophagy pathway is implicated in the LCSA-alleviated leaf senescence.

Here, the interactions between SA and MeJA in plant senescence were further investigated. By applying transcriptome and interaction network analysis, we identified autophagy-related (ATG) gene module. In contrast to wild type (Col-0), LCSA cannot alleviate the leaf yellowing phenotype in autophagy defective mutants upon MeJA 
treatment. Further results revealed that LCSA increased the number of autophagic bodies during MeJA-induced leaf senescence. Collectively, our work provides new insight that upregulation of autophagy by LCSA is a key regulator to alleviate MeJA-induced leaf senescence.

\section{MATERIALS AND METHODS}

\section{Plant materials and chemical treatments}

Arabidopsis plants of wild-type (WT, Col-0), atg5-1 (SAIL_129_B07), atg7-2 (GK-655B06) and eYFP-ATG8e (Zhuang et al., 2013) were grown in a greenhouse at $22{ }^{\circ} \mathrm{C}$ with $16 \mathrm{~h}$ light photoperiod $\left(120 \mu \mathrm{mol}\right.$ quanta $\left.{ }^{-2} \mathrm{~m}^{-2}\right)$. Phytohormones treatment was performed as described by Ji et al (2016). Briefly, the 3rd and 4th rosette leaves from four weeks of plants were detached and incubated in 3 mM MES buffer ( $\mathrm{pH}$ 5.8) containing $50 \mu \mathrm{M}$ methyl jasmonate (MeJA) and/or $10 \mu \mathrm{M}$ salicylic acid (SA). MeJA was prepared from a $50 \mathrm{mM}$ stock solution in ethanol. Solutions without MeJA were supplemented with equal amounts of ethanol. Concanamycin A (ConcA) was prepared as a $1 \mathrm{mM}$ stock solution in DMSO and used at final concentration $1 \mu \mathrm{M}$.

\section{Photochemical efficiency and chlorophyll content measurements}

The photochemical efficiency was measured with an Imaging-PAM Chlorophyll Fluorometer (PAM-MINI, Walz, Germany) followed the procedure described previously (Zhou et al., 2015). After dark-adapted for $1 \mathrm{~h}$, parameters Fo (minimum fluorescence with PSII reaction centers fully open) and Fm (maximum fluorescence after dark adaptation) were acquired with a $0.8-\mathrm{s}$ saturating pulse $\left(4,000 \mu \mathrm{mol}\right.$ photons $\left.\mathrm{m}^{-2} \mathrm{~s}^{-1}\right)$. The value of $\mathrm{Fv} / \mathrm{Fm}$ was calculated by the formulas $(\mathrm{Fm}-\mathrm{Fo}) / \mathrm{Fm}$. Total Chlorophyll was determined as reported by Coombs et al. (1987). Chlorophyll was extracted by immersion in $90 \%$ ethanol at $65{ }^{\circ} \mathrm{C}$ for $2 \mathrm{~h}$. The absorbance at $664 \mathrm{~nm}$ and $647 \mathrm{~nm}$ were determined with a Lambda 35 UV/VIS Spectrometer (Perkin-Elmer) (Zeng et al., 2016). The concentration per fresh weight of leaf tissue was calculated according to the formula: micromoles of chlorophyll per milliliter per gram fresh weight $=7.93(\mathrm{A664})+19.53(\mathrm{A647})$. The percentages of Fv/Fm and chlorophyll content are calculated relative to the initial levels of samples before treatment (time zero).

\section{RNA-Seq analysis}

Detached 3rd and 4th rosette leaves from 4-week old plants were immersed in $3 \mathrm{mM}$ MES buffer ( $\mathrm{pH}$ 5.8) containing $10 \mu \mathrm{M} \mathrm{SA}, 50 \mu \mathrm{M}$ MeJA, and MeJA together with SA for $24 \mathrm{~h}$. 
Total RNA for RNA-Seq was extracted from leaves using a Hipure plant RNA kit (Magen, China). Purified RNA was analyzed either using a ND-1000 Nanodrop (Thermo Fisher, USA), or by agarose gel electrophoresis to determine the RNA quantity. Those RNA samples with no smear seen on agarose gels, a 260/280 ratio above 2.0, and RNA integrity number greater than 8.0 were used. For RNA-Seq analysis, we mixed three replication samples for each treatment into one, and total RNA samples were then sent to RiboBio Co., Ltd (Guangzhou, China) for sequencing. The NEBNext Poly(A) mRNA Magnetic Isolation Module (NEB, USA) was used for mRNA purification. The Ultra II RNA Library Prep Kit for Illumina was used for RNA library construction. The libraries were sequenced as 50-bp single end reads using Illumina Hiseq2500 according to the manufacturer's instructions.

\section{Differential Expression Analysis}

Raw read count of each gene was generated using HTSeq with union-count mode (Love et al., 2014). After normalization by Reads Per Kilobase per Million mapped reads ( $r p k m)$, normalized read count table was used for determining differentially expressed genes (DEGs) (Anders and Huber, 2010; Cheng et al., 2016a, 2016b), which were defined as those with 2-fold changes. Fold change was calculated using log2 (normalized read count+1). An R package clusterprofiler was used to perform the functional category analysis to detect the significantly enriched Gene Ontology (GO) terms (Cheng and Leung, 2018a, and 2018b). Significantly enriched GO terms were selected by a threshold of $p \leq$ 0.05. Protein-protein interaction (PPI) data was obtained from the STRING database (v.10, http://string-db.org) (Szklarczyk et al., 2014). To construct a high-confidence network, only the PPIs with confidence scores larger than 0.7 were considered in this work. ClusterONE was adopted for the identification of protein clusters or functional modules using default parameters as described previously (Cheng et al., 2017; Cheng et al., 2019). The protein modules including five or more than five members and having connection density over 0.5 are defined as modules.

\section{RT-qPCR}

Total RNA was isolated using Eastep Super RNA Kit (Promega, Shanghai, China) and genomic DNA was removed using DNase I. $1 \mu \mathrm{g}$ of RNA was used to make cDNA with the GoScript $^{\mathrm{TM}}$ Reverse Transcription System (Promega, Shanghai, China). For qPCR $10 \mu \mathrm{L}$ of Green-2-Go 2X qPCR-S Mastermix (Sangon, Shanghai, China) and $1 \mu \mathrm{L}$ of cDNA (100 $\mathrm{ng} / \mu \mathrm{L}$ ) for a total of $20 \mu \mathrm{L}$ was used in each well. Real-time PCR was done on a CFX 
172 Connect Real-Time System (BioRad) at $95^{\circ} \mathrm{C}$ for 2 mins, and 45 cycles of $95^{\circ} \mathrm{C}$ for $15 \mathrm{~s}$,

$17355^{\circ} \mathrm{C}$ for $30 \mathrm{~s}$, and $72{ }^{\circ} \mathrm{C}$ for $30 \mathrm{~s}$ followed by a melting curve analysis. For each sample 3

174 biological reps were used and repeated 3 times for technical replication. qPCR was

175 analyzed using the $\Delta \Delta \mathrm{Ct}$ method. Primers for qPCR were showed in Table S2. Statistical

176 significance was determined using Duncan's multiple range test.

\section{Confocal microscopy}

178 Detached 3rd and 4th rosette leaves were immersed in $3 \mathrm{mM}$ MES buffer $(\mathrm{pH} 5.8)$ 179 containing $50 \mu \mathrm{M} \mathrm{MeJA}$ and/or $10 \mu \mathrm{M} \mathrm{SA}$ for $24 \mathrm{~h}$. ConcA $(1 \mu \mathrm{M})$ was added at $6 \mathrm{~h}$ before 180 confocal imaging. Confocal images were captured with 63x (numerical aperture [NA], 1.4) 181 objective using an LSM 880 microscope (Zeiss). For quantification of autophagic puncta, 182 randomly selected 15 to 20 images for each three independent experiments were 183 quantified with ImageJ. All images were collected with the same settings determined prior 184 to the experiment to yield nonsaturating conditions.

\section{RESULTS}

\section{LCSA delays MeJA-induced leaf senescence}

187

Our previous results indicated that SA delays MeJA-induced leaf senescence in a concentration-dependent manner, showing accelerated by high SA concentrations (greater than $100 \mu \mathrm{M})$ but attenuated by low SA concentrations (1-50 $\mu \mathrm{M})$ (Ji et al., 2016). On this basis, $10 \mu \mathrm{M} \mathrm{SA}$, the most effective concentration according to Ji et al., 2016, was selected as low working solution to further confirm the effect of LCSA. As shown in Figure 1, in contrast to control, LCSA did not appear to have a discernible effect on senescence. Leaves incubated with MeJA $(50 \mu \mathrm{M})$ were greatly turned yellow after 5 days treatment. However, when MeJA worked together with LCSA (MeJA+LCSA), the leaf yellowing was alleviated (Figure 1A). Consistent with the visible phenotype, the photochemical efficiency $\mathrm{Fv} / \mathrm{Fm}$ and loss of chlorophyll content in the leaves combined treatment with LCSA and MeJA was less severe relative to that of the leaves treated with MeJA alone (Figure 1B and $1 \mathrm{C}$ ). These physiological and biochemical data is consistent with our previous finding that LCSA provide protection against senescence caused by MeJA.

\section{Expression patterns of genes in LCSA-induced delayed leaf senescence}

To investigate the genome-wide effect of LCSA on MeJA-induced gene expression changes, we performed RNA-sequencing experiments. Since gene transcription regulation occurs prior to visible phenotype, leaves treatment with phytohormones at $1 \mathrm{~d}$ were 
selected according to our previous study (Ji et al., 2016). Totally, 408, 2536 and 2800 genes displayed at least 2-fold changes in the expression level of LCSA, MeJA, and MeJA+LCSA -treated leaves, respectively, relative to control leaves (Figure 2A). Of these, the number of differentially expressed genes (DEGs) of LCSA alone were greatly less than that in MeJA or MeJA+LCSA treatment group, in consistent with the inconspicuous phenotype between SA and control leaves (Figure 1). Therefore, our study is mainly concentrated on the differential expression of genes between MeJA and MeJA+LCSA.

To interpret the up-regulated and down-regulated DEGs resulting from the MeJA and MeJA+LCSA treatment, functional enrichment of Gene Ontology (GO) terms was performed using the hypergeometric test $(P$-value $<0.05)$. The analysis of biological process GO terms illustrated that most of the induced DEGs related to amino acid (Glutathione, Cyanamino acid, arginine, proline, alanine, aspartate and glutamate) metabolism, nitrogen metabolism, and flavonoid biosynthesis, whereas, the repressed DEGs mainly related to carbon metabolism, photosynthesis, and glycolysis (Figure 2B). These features of nitrogen and carbohydrate metabolism are consistent with the senescing phenotype of leaves. In contrast to MeJA alone, unexpectedly, LCSA together with MeJA treatment did not make much differences on the enriched biological processes (Figure 2C). The heatmap illustrated the top 50 up-regulated and down-regulated DEGs, which also revealed an extremely similar expression pattern between the DEGs of MeJA and MeJA+LCSA treatment (Figure 2D). These results indicated that LCSA does not appear to

\section{Network analysis identifies autophagy-related gene module}

Since the enrichment analysis only provided undifferentiated biological processes about basal metabolism, network analysis was conducted using DEGs that induced by MeJA and MeJA+LCSA, respectively. The protein-protein interactions (PPI) were collected from the STRING database, and only the PPIs with confidence scores higher than 0.7 were selected, resulting in a high confidence network with 719964 interactions and 17372 proteins. ClusterONE was used to identify functional protein modules, which were defined by the protein clusters including five or more than five members and having connection density over 0.5 (Cheng et al., 2019). According to such screening specifications, we identified 15 gene modules in MeJA treatment group and 16 gene modules in MeJA+LCSA group, respectively (Figure S1 and S2). Of these, six gene modules were specially detected in the 
induced seven gene modules, covering genes involved in autophagy-related pathway, phytohormone response, ATP-binding cassette transporters, aquaporins, and flavonoid biosynthesis (Figure 3A). In this context, autophagy is an essential intracellular degradation system that plays important roles in nutrient remobilization during leaf senescence (AvilaOspina et al., 2014). We found that the transcript abundance for ATG proteins (ATG4, ATG8, ATG9, and ATG12) was differentially sensitive to the MeJA+LCSA treatment. From the enriched biological processes and molecular functions, we observed that these ATGs are the core components that contribute to autophagosome mature and biogenesis (Figure 3B and $3 \mathrm{C}$ ). Collectively, these results suggest a framework in which MeJA together with LCSA regulates the abundance of specific gene network, such as the autophagy process.

We next investigated whether the autophagy pathway was involved in LCSA-delayed leaf senescence. Ten ATG genes (ATG4A, ATG4B, ATG5, ATG6, ATG7, ATG8A, ATG8E, ATG8H, ATG12A, and ATG12B) that implemented in autophagosome formation were examined by RT-qPCR (Figure 4). In contrast to MeJA alone, most of these determined ATG genes, except for ATG8A and ATG8E, were up-regulated by the combined treatment group (MeJA+LCSA). The differential gene expression of ATG8 isoforms is possible due to they have different expression pattern in distinct tissues (Hanaoka et al., 2002). Interestingly, it should be mentioned that MeJA together with LCSA did not stimulate a much more increase in gene expression compared with control, especially LCSA treatment (Figure 4). These results indicate that restoration of ATG genes expression is closely related to LCSA-delayed leaf senescence.

\section{SA-delayed leaf senescence is dependent on a functional autophagy pathway}

To further resolve whether autophagy pathway was crucial for LCSA-delayed leaf senescence, two autophagy defective mutants (atg5-1 and atg7-2), that involved in ATG8 lipidation during phagophore elongation (Feng et al., 2014), were analyzed upon LCSA and/or MeJA treatment. In contrast to wild type (Col-0), leaves from atg5-1 and atg7-2 mutants were showed much more yellowing after incubated with MeJA for 5 days (Figure $5 A)$. As expect, the leaf yellowing phenotype was not alleviated when MeJA worked together with LCSA (Figure 5A). Consistently, the photochemical efficiency Fv/Fm in the atg5-1 and atg7-2 mutant leaves treated with MeJA+LCSA was not restored relative to that of the leaves treated with MeJA (Figure 5B). Similarly, none of the two mutants had recovered relative chlorophyll content as the Col-0 after combined treatment with MeJA and LCSA (Figure 5C). These genetic results clearly illustrated that the protection against MeJA-induced senescence by LCSA is dependent on a functional autophagy pathway. 


\section{SA increases autophagy activity upon MeJA-induced leaf senescence} Since autophagy pathway was verified involved in LCSA-delayed leaf senescence, we next further determined the detailed autophagy activity. Wild-type Arabidopsis plants expressing the eYFP-ATG8e fusion protein were subjected to LCSA and/or MeJA treatment, and the effects of LCSA on autophagy activity were analyzed by confocal microscopy of the YFP fluorescence. In control and LCSA treatment conditions, we observed a few fluorescent punctate structures that were identified previously as ATG8-tagged autophagosomes (or autophagic bodies) (Zhuang et al., 2013). Incubation of MeJA alone induced a slightly increase in accumulation of autophagic bodies (Figure 6A and 6B). However, when the detached leaves were subjected to combined treatment with MeJA and LCSA, there was a greatly increase in the fluorescent vesicles (Figure 6A and 6B). The statistical results showed that the number of autophagic bodies was more than 2-fold higher in MeJA+LCSA group than that of treatment with MeJA alone (Figure 6C and 6D). Taken together, our observations collectively suggest that LCSA activates the autophagy activity to delay MeJA-induced leaf senescence.

\section{DISCUSSION}

As the final stage of leaf development, leaf senescence is a complex process that involves thousands of genes and multiple layers of regulation. Mechanisms governing the specificity regulation of phytohormones and output gene expression are therefore of great interest. The primary objective of the work is to further explore the crosstalk between SA and JA signaling in regulating plant leaf senescence. We have concentrated on examining the mechanisms likely to underpin changes in the transcriptome in response to LCSA and/or MeJA. Specifically, an autophagy module was identified from the DEGs that exclusively induced by MeJA together with SA (Figure 3). Further results demonstrate that the upregulation of autophagy by LCSA serves important function in alleviating MeJA-induced leaf senescence (Figure 5 and 6).

Previously, we found that SA delays MeJA-induced leaf senescence in a concentration dependent manner ( $\mathrm{Ji}$ et al, 2016). The dosage-dependent effect of SA also has been reported in plant root meristem regulation. SA at low levels (below $50 \mu \mathrm{M}$ ) promotes adventitious roots and alters architecture of the root apical meristem, whereas highconcentration SA $(>50 \mu \mathrm{M})$ inhibits root growth (Pasternak et al., 2019). Such discrepancies are probably due to SA acts as a developmental regulator at low levels, but acts as a stress hormone at high levels (Pasternak et al., 2019). Interestingly, RNA-Seq 
results showed that the number of DEGs in LCSA alone treatment were less than MeJA or LCSA and MeJA combined treatment group (Figure 2A), which consistent with LCSA itself did not have a discernible effect on senescence, showing the inconspicuous phenotype between LCSA and control leaves (Figure 1). Moreover, in contrast to MeJA alone, LCSA together with MeJA treatment did not make much differences on the biological process of GO terms (Figure $2 \mathrm{C}$ ). These results indicated that LCSA at low level is more likely function as a signaling regulator, which does not have a marked impact on the basal metabolism at least at the genetic regulatory level.

Autophagy promotes cell survival by adapting cells to stress conditions both in plants and mammals. Recent reverse-genetic studies have revealed that autophagy is closely associated with plant senescence, and autophagy defective mutants like atg2, atg5 and atg7 all showed early yellowing leaf symptoms (Doelling et al., 2002; Yoshimoto et al., 2009). SA is one of the most promising phytohormones that contribute to the induction of autophagy under stress. It has previously been reported that autophagy negatively regulates cell death by controlling NPR1-dependent SA signaling during senescence in Arabidopsis (Yoshimoto et al., 2009). Here, the ClusterONE was applied to identify discrete gene modules based on PPI network. We identified several modules including autophagyrelated network in DEGs that exclusively induced by MeJA together with LCSA (Figure 3A). Importantly, the protection against MeJA-induced senescence by LCSA was abolished in autophagy defective mutant atg5-1 and atg7-2 (Figure 5). These data strongly suggest an important role for autophagy in LCSA-alleviated leaf senescence. Notably, unlike the greatly increase of autophagic bodies induced by MeJA+LCSA, autophagosomes under LCSA alone treatment were not statistically significant when compared with control (Figure 6). Nevertheless, it is worth pointing out that $S A$ at $100 \mu \mathrm{M}$, a high-concentration that could promote leaf senescence based on our previous study (Chai et al., 2016), greatly induced autophagic structures formation (Figure S4). In this context, we speculate that LCSA might be function like a priming regulator, which could initiate signal amplification and lead to a robust activation of stress response upon MeJA treatment. Actually, the priming induced by some plant activators (e.g. $\beta$-aminobutyric acid, and thiamine) are dependent on SA signaling (Ahn et al., 2005; Jung et al., 2009; Zhou et al., 2013). It would be interesting to test the priming effect of LCSA on leaf senescence in future research.

In summary, this study further investigated the interactions between SA and MeJA in plant senescence. Several modules including an autophagy-related (ATG) cluster were identified by analyzing the transcriptome data and protein interaction networks. Further 
results showed that LCSA could upregulate autophagy to alleviate MeJA-induced leaf senescence. This was confirmed by founding that LCSA cannot alleviate the leaf yellowing phenotype in autophagy defective mutants upon MeJA treatment. Collectively, our work reveals LCSA tend to function as a signaling regulator to upregulate autophagy pathway, which serves as an important cellular mechanism responsible for alleviation of MeJAinduced leaf senescence.

\section{Data availability}

RNA-seq data were deposited in the Sequence Read Archive (SRA) database https://www.ncbi.nlm.nih.gov/sra with accession no. PRJNA578602.

\section{AUTHOR CONTRIBUTIONS}

$\mathrm{JZ}$ and $\mathrm{LC}$ designed the research. RY, JY, and YJ conducted the experiments. RY, XL, JL, JZ and LC analyzed data. JZ and LC wrote the manuscript. All authors read and approved the manuscript.

\section{ACKNOWLEDGEMENTS}

Thanks for Professor Liwen Jiang (the Chinese University of Hong Kong) for giving the Arabidopsis seeds materials atg5-1, atg7-2 and eYFP-ATG8e. Thanks for Yang Lv (Fengyuan biotechnology co. LTD, Shanghai, China) for the valuable suggestions for this manuscript. This work was supported by National Science Foundation of China (NSFC) (31600288); Guangdong Provincial Science and Technology Project (2016A020210127); SCNU Youth Teacher Research and Development Fund Project (671075); Scientific Research Projects of Guangzhou (201805010002).

\section{CONFLICTS OF INTEREST}

The authors declare no conflict of interest. 


\section{REFERENCES}

Anders, S., Huber, W. (2010). Differential expression analysis for sequence count data. Genome Biol. 11(10), R106. doi: 10.1186/gb-2010-11-10-r106

Ahn, I. P., Kim, S., Lee, Y. H., Suh, S. C. (2007). Vitamin B1-induced priming is dependent on hydrogen peroxide and the NPR1 gene in Arabidopsis. Plant Physiol. 143(2), 838-848. doi: 10.1104/pp.106.092627

Avila-Ospina, L., Moison, M., Yoshimoto, K., Masclaux-Daubresse, C. (2014). Autophagy, plant senescence, and nutrient recycling. J. Exp. Bot. 65(14), 3799-3811. doi: 10.1093/jxb/eru039

Bhattacharjee, S. (2005). Reactive oxygen species and oxidative burst: roles in stress, senescence and signal transducation in plants. Curr. Sci. 89, 1113-1121.

Chai, J., Liu, J., Zhou, J., Xing, D. (2014). Mitogen-activated protein kinase 6 regulates NPR1 gene expression and activation during leaf senescence induced by salicylic acid. J. Exp. Bot. 65(22), 6513-6528. doi: 10.1093/jxb/eru369

Cheng, L., \& Leung, K. S. (2018a). Identification and characterization of moonlighting long non-coding RNAs based on RNA and protein interactome. Bioinformatics, 34(20), 3519-3528. doi: 10.1093/bioinformatics/bty399

Cheng, L., \& Leung, K. S. (2018b). Quantification of non-coding RNA target localization diversity and its application in cancers. J Mol. Cell Biol. 10(2), 130-138. doi: 10.1093/jmcb/mjy006

Cheng, L., Liu, P., Leung, K. S. (2017). SMILE: a novel procedure for subcellular module identification with localisation expansion. IET Syst. Biol. 12(2), 55-61. doi: 10.1049/iet-syb.2017.0085

Cheng, L., Liu, P., Wang, D., Leung, K. S. (2019). Exploiting locational and topological overlap model to identify modules in protein interaction networks. BMC bioinformatics, 20(1), 23. doi: 10.1186/s12859-019-2598-7

Cheng, L., Lo, L. Y., Tang, N. L., Wang, D., \& Leung, K. S. (2016a). CrossNorm: a novel normalization strategy for microarray data in cancers. Sci. Rep. 6, 18898. doi: 10.1038/srep18898

Cheng, L., Wang, X., Wong, P. K., Lee, K. Y., Li, L., Xu, B., et al. (2016b). ICN: a normalization method for gene expression data considering the over-expression of informative genes. Mol. BioSyst. 12(10), 3057-3066. doi: 10.1039/c6mb00386a

Coombs. J., G. Hind, R. C. Leegood, L. L. Tieszen and A. Vonshak (1987). Analytical Techniques. In: Techniques in Bioproductivity and photosynthesis 2nd Edition. (Eds) J. Coombs, D. O. Hall, S. P. Long and J. M. O. Scurlock. 219-220. Pergamon Press.

Doelling, J. H., Walker, J. M., Friedman, E. M., Thompson, A. R., Vierstra, R. D. (2002). The APG8/12-activating enzyme APG7 is required for proper nutrient recycling and senescence in Arabidopsis thaliana. J. Biol. Chem. 277(36), 33105-33114.

Feng, Y., He, D., Yao, Z., \& Klionsky, D. J. (2014). The machinery of macroautophagy. Cell Res. 24(1), 24-41. doi: 10.1038/cr.2013.168

Gan S, Amasino RM. 1995. Inhibition of leaf senescence by autoregulated production of cytokinin. Science 270:1986-1988. doi: 10.1126/science.270.5244.1986

Ji, Y., Liu, J., Xing, D. (2016). Low concentrations of salicylic acid delay methyl jasmonate-induced leaf senescence by up-regulating nitric oxide synthase activity. J. 
Exp. Bot. 67(17), 5233-5245. doi: 10.1093/jxb/erw280

Jung, H. W., Tschaplinski, T. J., Wang, L., Glazebrook, J., Greenberg, J. T. (2009).

Priming in systemic plant immunity. Science, 324(5923), 89-91. doi:

10.1126/science. 1170025

Hanaoka, H., Noda, T., Shirano, Y., Kato, T., Hayashi, H., Shibata, D., et al. (2002). Leaf senescence and starvation-induced chlorosis are accelerated by the disruption of an Arabidopsis autophagy gene. Plant Physiol. 129(3), 1181-1193. doi:

10.1104/pp.011024

He, Y., Fukushige, H., Hildebrand, D.F., Gan, S. 2002. Evidence supporting a role of jasmonic acid in Arabidopsis leaf senescence. Plant Physiol. 128(3), 876-884. doi: 10.1104/pp.010843

Hung, K.T., Kao, C.H. 2004. Hydrogen peroxide is necessary for abscisic acid-induced senescence of rice leaves. J. Plant Physiol. 161(12), 1347-1357. doi: 10.1016/j.jplph.2004.05.011

Li, F., Chung, T., Vierstra, R. D. (2014). AUTOPHAGY-RELATED11 plays a critical role in general autophagy-and senescence-induced mitophagy in Arabidopsis. Plant Cell 26(2), 788-807. doi: 10.1105/tpc. 113.120014

Love, M. I., Huber, W., Anders, S. (2014). Moderated estimation of fold change and dispersion for RNA-seq data with DESeq2. Genome Biol. 15(12), 550. doi: 10.1186/s13059-014-0550-8

Lim, P.O., Woo, H.R., \& Nam, H.G. (2003). Molecular genetics of leaf senescence in Arabidopsis. Trends Plant Sci. 8(6), 272-278. doi: 10.1016/S1360-1385(03)00103-1

Lim, P.O., Kim, H.J., Nam, H.G. 2007. Leaf senescence. Annu. Rev. Plant Biol. 58, 115136. doi: 10.1146/annurev.arplant.57.032905.105316

Masclaux, C., Valadier, M.H., Brugiere, N., Morot-Gaudry, J.F., Hirel, B. 2000. Characterization of the sink/source transition in tobacco (Nicotiana tabacum L.) shoots in relation to nitrogen management and leaf senescence. Planta 211, 510518. doi: $10.1007 / \mathrm{s} 004250000310$

Miao Y., Zentgraf U. 2007. The antagonist function of Arabidopsis WRKY53 and ESR/ESP in leaf senescence is modulated by the jasmonic and salicylic acid equilibrium. Plant Cell 19, 819-830. doi: 10.1105/tpc.106.042705

Morris, K., Mackerness, S.A., Page, T., John, C.F., Murphy, A.M., et al. 2000. Salicylic acid has a role in regulating gene expression during senescence. Plant J. 23:677685. doi: 10.1046/j.1365-313x.2000.00836.x

Pasternak, T., Groot, E. P., Kazantsev, F. V., Teale, W., Omelyanchuk, N., Kovrizhnykh, V., et al. (2019). Salicylic acid affects root meristem patterning via auxin distribution in a concentration-dependent manner. Plant Physiol. 180(3), 1725-1739. doi: 10.1104/pp.19.00130

Quirino, B.F., Noh, Y.S., Himelblau, E., Amasino, R.M. (2000). Molecular aspects of leaf senescence. Trends Plant Sci. 5(7), 278-282. doi: 10.1016/S1360-1385(00)01655-1

Schippers, J.H. (2015). Transcriptional networks in leaf senescence. Curr. Opin. Plant Biol. 27, 77-83. doi: 10.1016/j.pbi.2015.06.018

Szklarczyk, D., Franceschini, A., Wyder, S., Forslund, K., Heller, D., Huerta-Cepas, J., et al. (2014). STRING v10: protein-protein interaction networks, integrated over the 
tree of life. Nucleic Acids Res. 43(1), 447-452. doi: 10.1093/nar/gku1003

Thaler, J. S., Humphrey, P. T., \& Whiteman, N. K. (2012). Evolution of jasmonate and salicylate signal crosstalk. Trends Plant Sci. 17(5), 260-270. doi:

10.1016/j.tplants.2012.02.010

van der Graaff, E., Schwacke, R., Schneider, A., Desimone, M., Flugge, U.I., Kunze, R. 2006. Transcription analysis of Arabidopsis membrane transporters and hormone pathways during developmental and induced leaf senescence. Plant Physiol. 141:776-792. doi: 10.1104/pp.106.079293

Xiong, Y., Contento, A. L., Bassham, D. C. (2005). AtATG18a is required for the formation of autophagosomes during nutrient stress and senescence in Arabidopsis thaliana. Plant J. 42(4), 535-546. doi: 10.1111/j.1365-313X.2005.02397.x

Xiong, Y., Contento, A. L., Nguyen, P. Q., Bassham, D. C. (2007). Degradation of oxidized proteins by autophagy during oxidative stress in Arabidopsis. Plant Physiol. 143(1), 291-299. doi: 10.1104/pp.106.092106

Yoshida, S. (2003). Molecular regulation of leaf senescence. Curr. Opin. Plant Biol. 6(1), 79-84. doi: 10.1016/S1369526602000092

Yoshimoto, K., Jikumaru, Y., Kamiya, Y., Kusano, M., Consonni, C., Panstruga, R., et al. (2009). Autophagy negatively regulates cell death by controlling NPR1-dependent salicylic acid signaling during senescence and the innate immune response in Arabidopsis. Plant Cell, 21(9), 2914-2927. doi: 10.1105/tpc.109.068635

Yue, H., Nie, S., Xing, D. (2012). Over-expression of Arabidopsis Bax inhibitor-1 delays methyl jasmonate-induced leaf senescence by suppressing the activation of MAP kinase 6. J. Exp. Bot. 63(12), 4463-4474. doi: 10.1093/jxb/ers122

Zeng, L., Wang, Y., Zhou, J. (2016). Spectral analysis on origination of the bands at 437 $\mathrm{nm}$ and $475.5 \mathrm{~nm}$ of chlorophyll fluorescence excitation spectrum in Arabidopsis chloroplasts. Luminescence, 31(3), 769-774. doi: 10.1002/bio.3022

Zhou, J., Sun, A., Xing, D. (2013). Modulation of cellular redox status by thiamineactivated NADPH oxidase confers Arabidopsis resistance to Sclerotinia sclerotiorum. J. Exp. Bot. 64(11), 3261-3272. doi: 10.1093/jxb/ert166

Zhou, J., Zeng, L., Liu, J., Xing, D. (2015). Manipulation of the xanthophyll cycle increases plant susceptibility to Sclerotinia sclerotiorum. PLoS Pathog. 11(5), e1004878. doi: 10.1371/journal.ppat.1004878

Zhuang, X., Wang, H., Lam, S. K., Gao, C., Wang, X., Cai, Y., Jiang, L. (2013). A BARdomain protein SH3P2, which binds to phosphatidylinositol 3-phosphate and ATG8, regulates autophagosome formation in Arabidopsis. Plant Cell, 25(11), 4596-4615. doi: $10.1105 /$ tpc. 113.118307 
Figure 1. LCSA alleviates MeJA-induced leaf senescence. (A) Phenotypes of detached leaves under LCSA and/or MeJA treatments. The 3rd and 4th rosette leaves were incubated in $3 \mathrm{mM}$ MES buffer ( $\mathrm{pH}$ 5.8) containing LCSA $(10 \mu \mathrm{M})$ or MeJA $(50 \mu \mathrm{M})$ alone or in combination (MeJA+LCSA) under continuous light for $5 \mathrm{~d}$. (B and $C$ ) Measurement of the maximum quantum efficiency of photosystem II (PSII) photochemistry (Fv/Fm) (B) and total chlorophyll content $(\mathrm{C})$ after LCSA and/or MeJA treatments. The percentages of Fv/Fm and chlorophyll content are relative to the initial levels at time zero. Data were the mean $\pm \mathrm{SE}$ of three independent experiments. Different letters indicate statistically significant differences between each treatment (Duncan's multiple range test, $p<0.05$ ).

Figure 2. RNA-Seq analyses of differentially expressed genes (DEGs) in samples treated with LCSA, MeJA and LCSA+MeJA. (A) Venn diagram showing the overlap of DEGs between LCSA, MeJA and LCSA+MeJA-treated samples. (B and C) The pathway enrichment analysis of up or down -regulated DEGs induced by MeJA alone (B) or regulated DEGs between MeJA and MeJA+LCSA treatment group.

Figure 3. Network analysis identifies distinct signal modules in the DEGs exclusively induced by LCSA+MeJA treatment. (A) Interconnected clusters enriched among the 889 genes and their interactions with neighboring genes. The autophagy specific module was drawn in a red dotted line. Genes are colored in red if they are induced and in blue if they are repressed. (B and C) Biological process (B) and molecular function (C) classification in gene ontology analysis of the DEGs that identified in coexpression networks.

Figure 4. RT-qPCR confirmation of differentially expressed genes that involved in regulation of autophagy. The relative mRNA expression levels were calculated using the $\Delta \Delta \mathrm{Ct}$ method. The value of each ATG genes were relative to the initial levels at time zero of treatment. Data were the mean \pm SE of three independent experiments. Different letters in each genes indicate statistically significant differences between the treatments (Duncan's multiple range test, $p<0.05$ ).

Figure 5. Defective in autophagy restrains the effect of SA on the senescence symptoms. (A) Phenotypes of LCSA-alleviated senescence in Col-0 and autophagy defective mutants (atg5-1 and atg7-2). Detached leaves form four-week-old Col-0, atg5-1, and atg7-2 plants were transferred to MES buffer ( $\mathrm{pH} 5.8)$ containing LCSA (10 $\mu \mathrm{M})$ or MeJA $(50 \mu \mathrm{M})$ or both MeJA and LCSA under continuous light and photographs were taken after 5 days of treatment. ( $B$ and $C$ ) Relative Fv/Fm (B) chlorophyll levels $(C)$ in the leaves of the Col-0, atg5-1, and atg7-2 described in (A). Data were the mean \pm SE of three independent experiments. Different letters indicate statistically significant differences between the treatments (Duncan's multiple range test, $p<0.05$ ).

Figure 6. LCSA enhances the formation of autophagosomes upon MeJA-induced leaf senescence. (A) Microscopic analyses of autophagosome-related structures in the mesophyll cells of eYFP-ATG8e plant under LCSA or MeJA or both MeJA and LCSA treatment. (B) Examination of autophagic bodies accumulated in the vacuoles. ConcA, 
403

404

405

406

407

408 concanamycin A. Bars, $20 \mu \mathrm{m}$. (C and D) Statistical analysis of the puncta numbers displayed in (A) and (B), respectively. The number of puncta was calculated per $0.01 \mathrm{~mm}^{2}$ from at least 15 pictures. This experiment was repeated in triplicate with similar results. Different letters indicate statistically significant differences between the treatments (Duncan's multiple range test, $p<0.05$ ). 

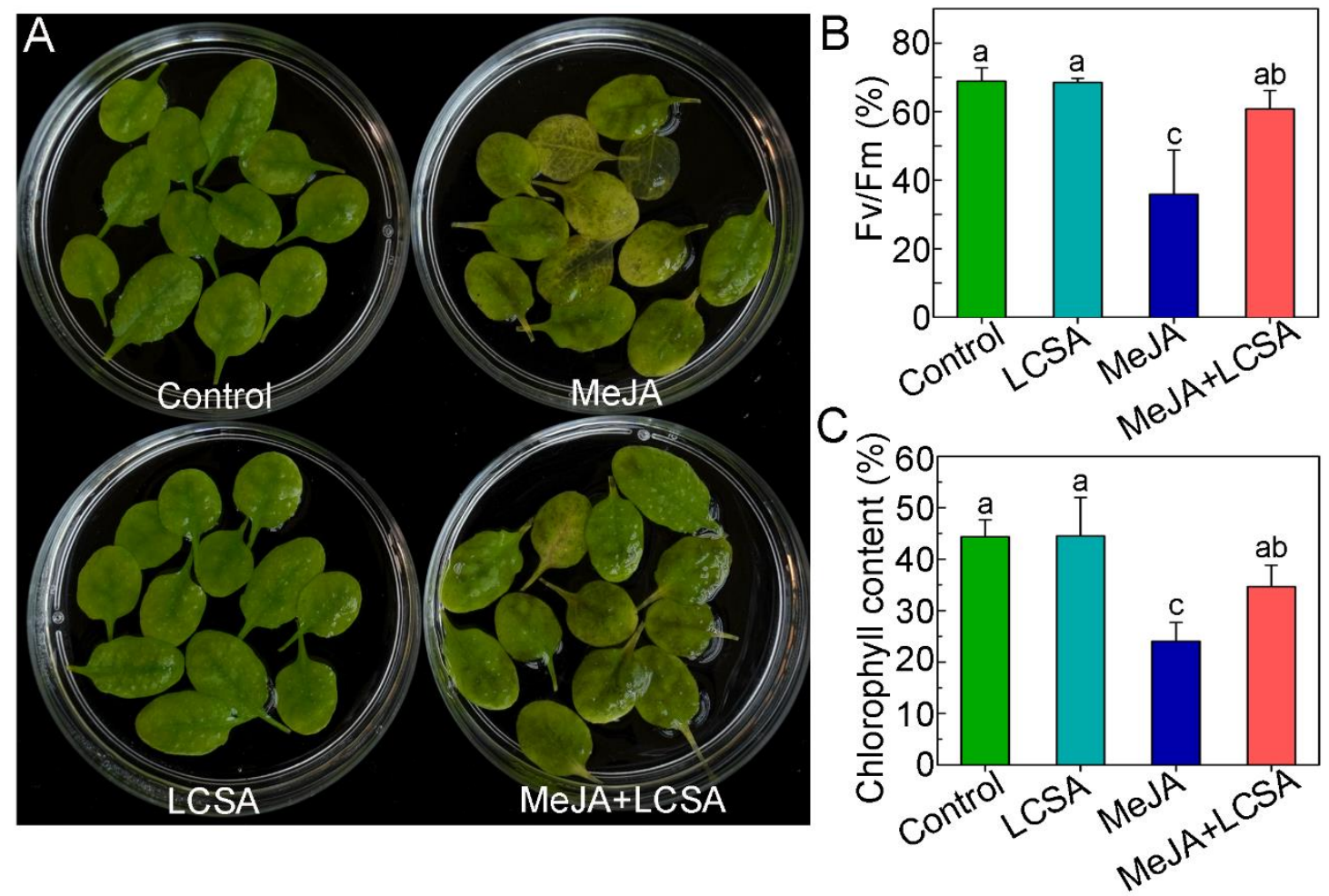

410 Figure 1. LCSA alleviates MeJA-induced leaf senescence. (A) Phenotypes of detached 411 leaves under LCSA and/or MeJA treatments. The 3rd and 4th rosette leaves were 412 incubated in $3 \mathrm{mM}$ MES buffer $(\mathrm{pH} 5.8)$ containing LCSA $(10 \mu \mathrm{M})$ or MeJA $(50 \mu \mathrm{M})$ alone 413 or in combination (MeJA+LCSA) under continuous light for $5 \mathrm{~d}$. (B and C) Measurement of 414 the maximum quantum efficiency of photosystem II (PSII) photochemistry (Fv/Fm) (B) and total chlorophyll content (C) after LCSA and/or MeJA treatments. The percentages of $\mathrm{Fv} / \mathrm{Fm}$ and chlorophyll content are relative to the initial levels at time zero. Data were the mean \pm SE of three independent experiments. Different letters indicate statistically significant differences between each treatment (Duncan's multiple range test, $p<0.05$ ). 
A Up-regulated DEGs
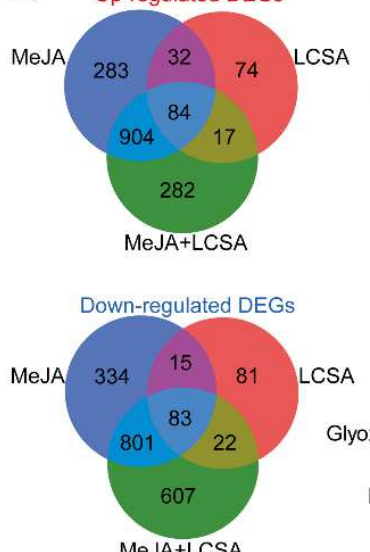

MeJA+LCSA

D

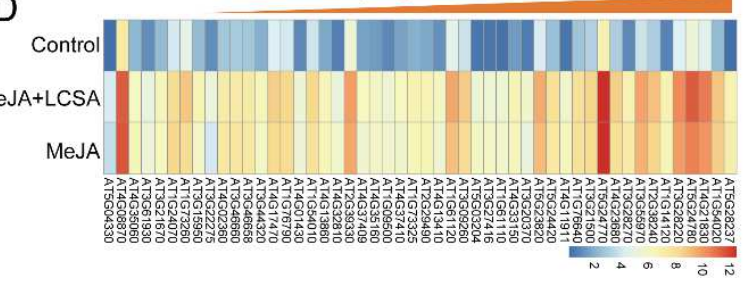

Gin metabolism

Cyanoamino acid metabolism. alpha-Linolenic acid metabolism Arg and Pro metabolism.

Ala, Asp, and Glu metabolism . Nitrogen metabolism

Carbon metabolism

Glycolysis/Gluconeogenesis . Carbon fixation

oxylate, dicarboxylate metabolism

Photosynthesis

Photosynthesis-antenna proteins
MeJA up-regulated DEGs Flavonoid biosynthesis .

$$
\begin{array}{r}
0_{03}^{0.05} 0.01 \\
\text { GeneRatio }
\end{array}
$$

MeJA down-regulated DEGs

$$
0.040 .080 .120 .16
$$

GeneRatio
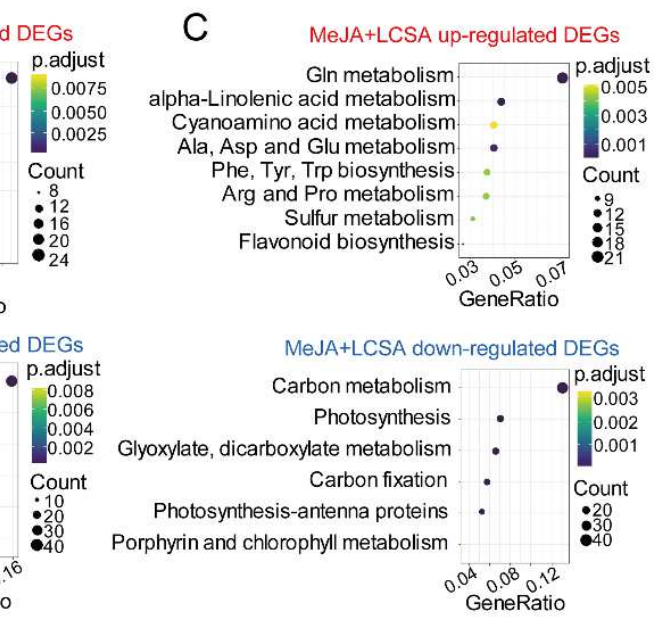

MeJA+LCSA down-regulated DEGs

Carbon metabolism p.adjust Photosynthesis ylate metabolism

Carbon fixation

Photosynthesis-antenna proteins

Porphyrin and chlorophyll metabolism

$$
\begin{aligned}
& 0.040 .0 .12 \\
& \text { GeneRatio }
\end{aligned}
$$

Down-regulated DEGs

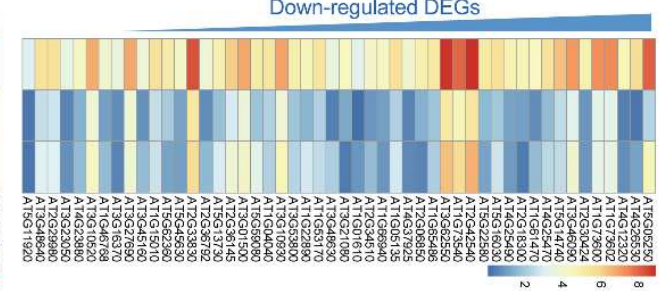

Figure 2. RNA-Seq analyses of differentially expressed genes (DEGs) in samples treated with LCSA, MeJA and LCSA+MeJA. (A) Venn diagram showing the overlap of DEGs between LCSA, MeJA and LCSA+MeJA-treated samples. (B and C) The pathway enrichment analysis of up or down -regulated DEGs induced by MeJA alone (B) or LCSA+MeJA (C). (D) The heatmap showing expression of top 50 up-regulated and downregulated DEGs between MeJA and MeJA+LCSA treatment group. 

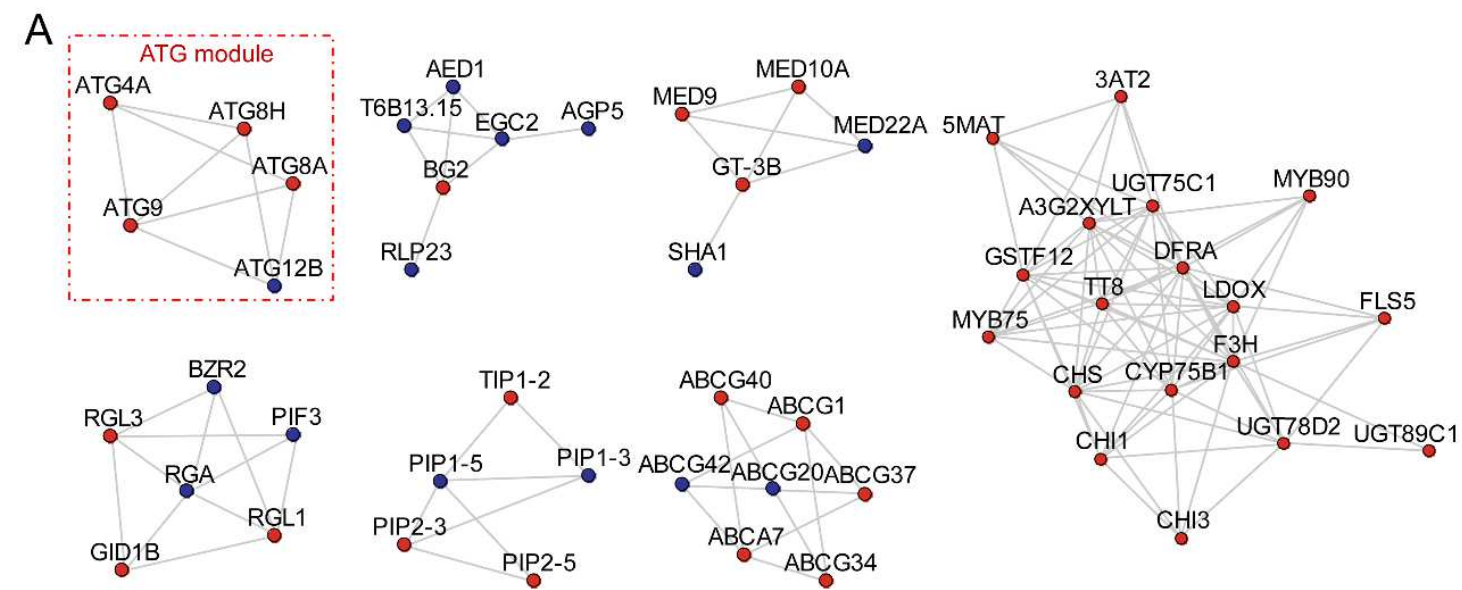

B
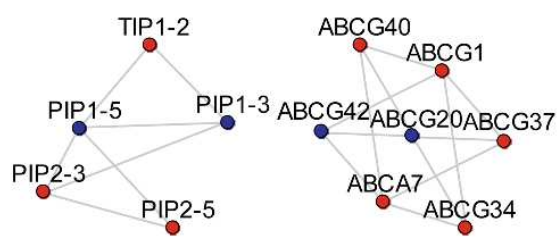

C

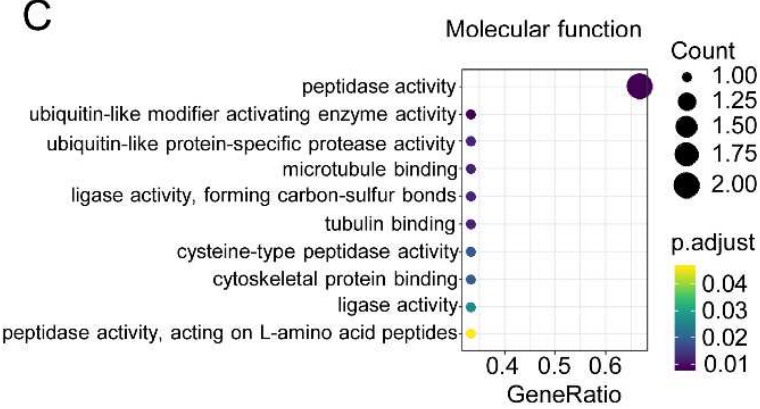

Figure 3. Network analysis identifies distinct signal modules in the DEGs exclusively induced by LCSA+MeJA treatment. (A) Interconnected clusters enriched among the 889 genes and their interactions with neighboring genes. The autophagy specific module was drawn in a red dotted line. Genes are colored in red if they are induced and in blue if they are repressed. (B and C) Biological process (B) and molecular function $(C)$ classification in gene ontology analysis of the DEGs that identified in coexpression networks. 


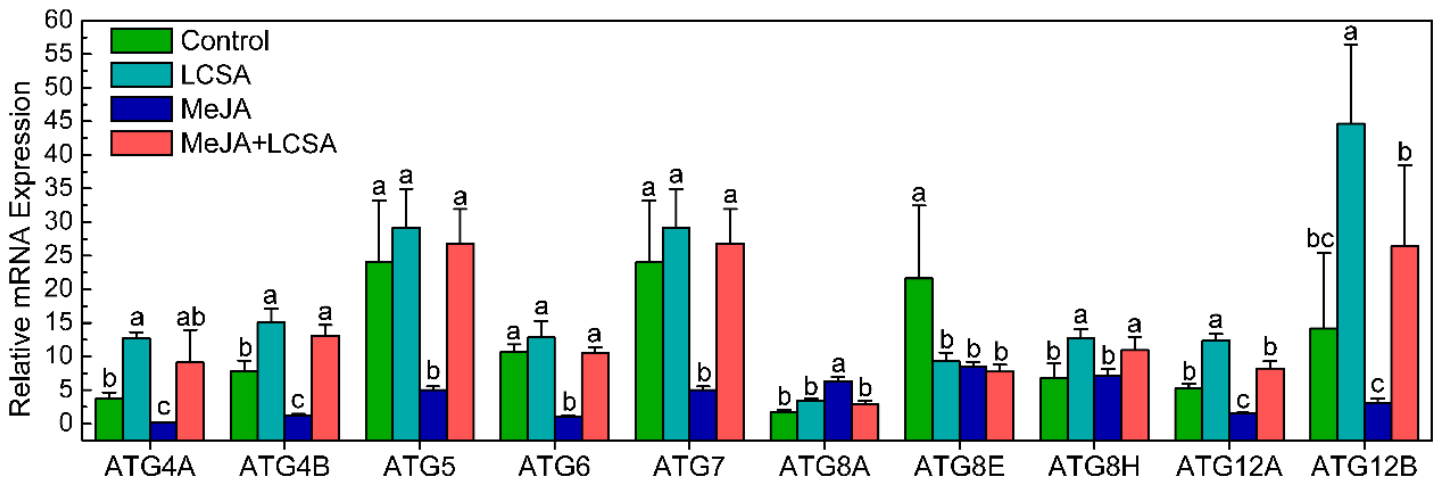

Figure 4. RT-qPCR confirmation of differentially expressed genes that involved in regulation of autophagy. The relative mRNA expression levels were calculated using the $\triangle \triangle \mathrm{Ct}$ method. The value of each ATG genes were relative to the initial levels at time zero of treatment. Data were the mean \pm SE of three independent experiments. Different letters in each genes indicate statistically significant differences between the treatments (Duncan's multiple range test, $p<0.05$ ). 

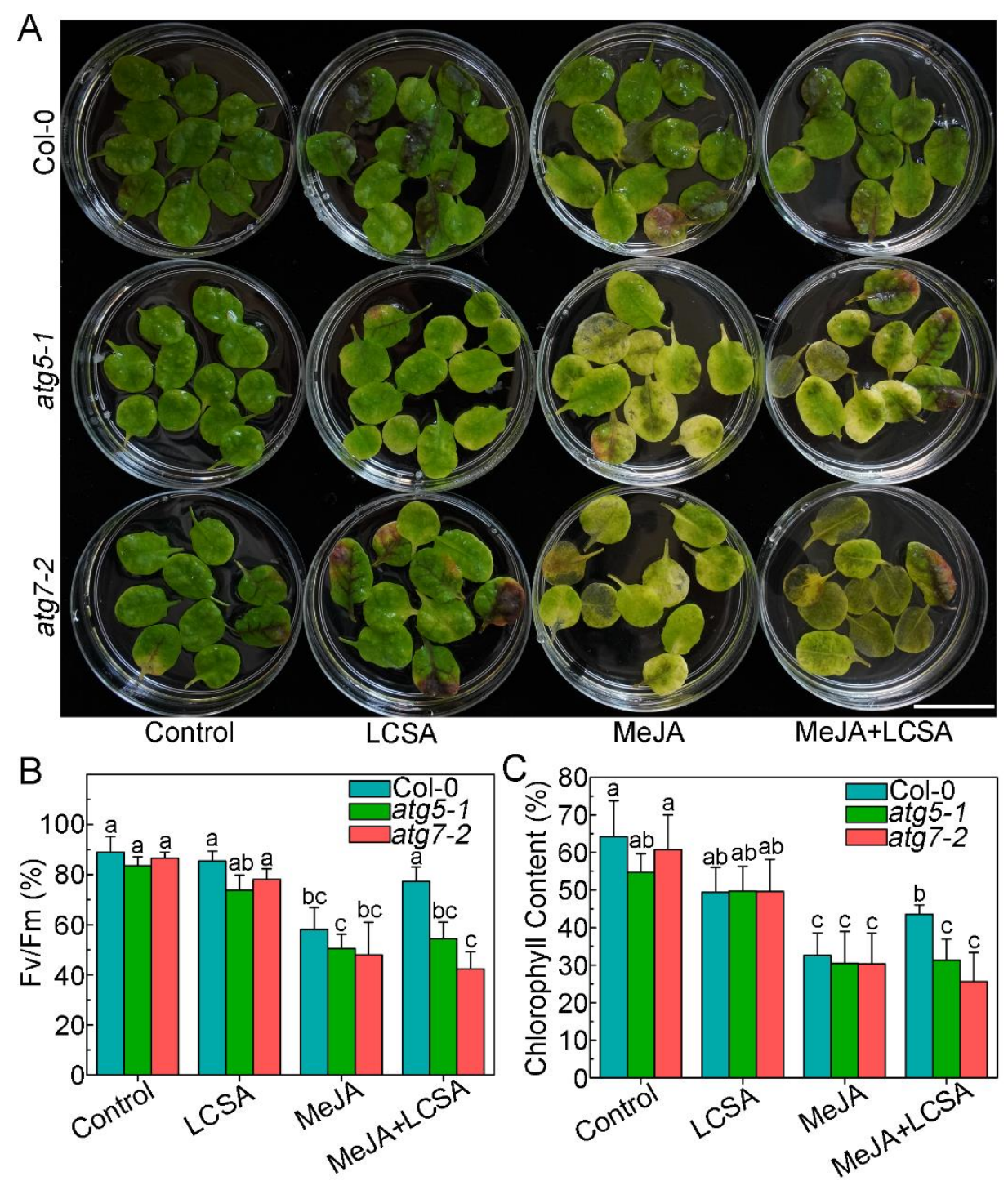

446 Figure 5. Defective in autophagy restrains the effect of SA on the senescence

447 symptoms. (A) Phenotypes of LCSA-alleviated senescence in Col-0 and autophagy 448 defective mutants (atg5-1 and atg7-2). Detached leaves form four-week-old Col-0, atg5-1, 449 and atg7-2 plants were transferred to MES buffer ( $\mathrm{pH} 5.8)$ containing LCSA (10 $\mu \mathrm{M})$ or 450 MeJA $(50 \mu \mathrm{M})$ or both MeJA and LCSA under continuous light and photographs were taken 451 after 5 days of treatment. (B and $C$ ) Relative Fv/Fm (B) chlorophyll levels (C) in the leaves 452 of the Col-0, atg5-1, and atg7-2 described in (A). Data were the mean \pm SE of three 453 independent experiments. Different letters indicate statistically significant differences 454 between the treatments (Duncan's multiple range test, $p<0.05$ ). 

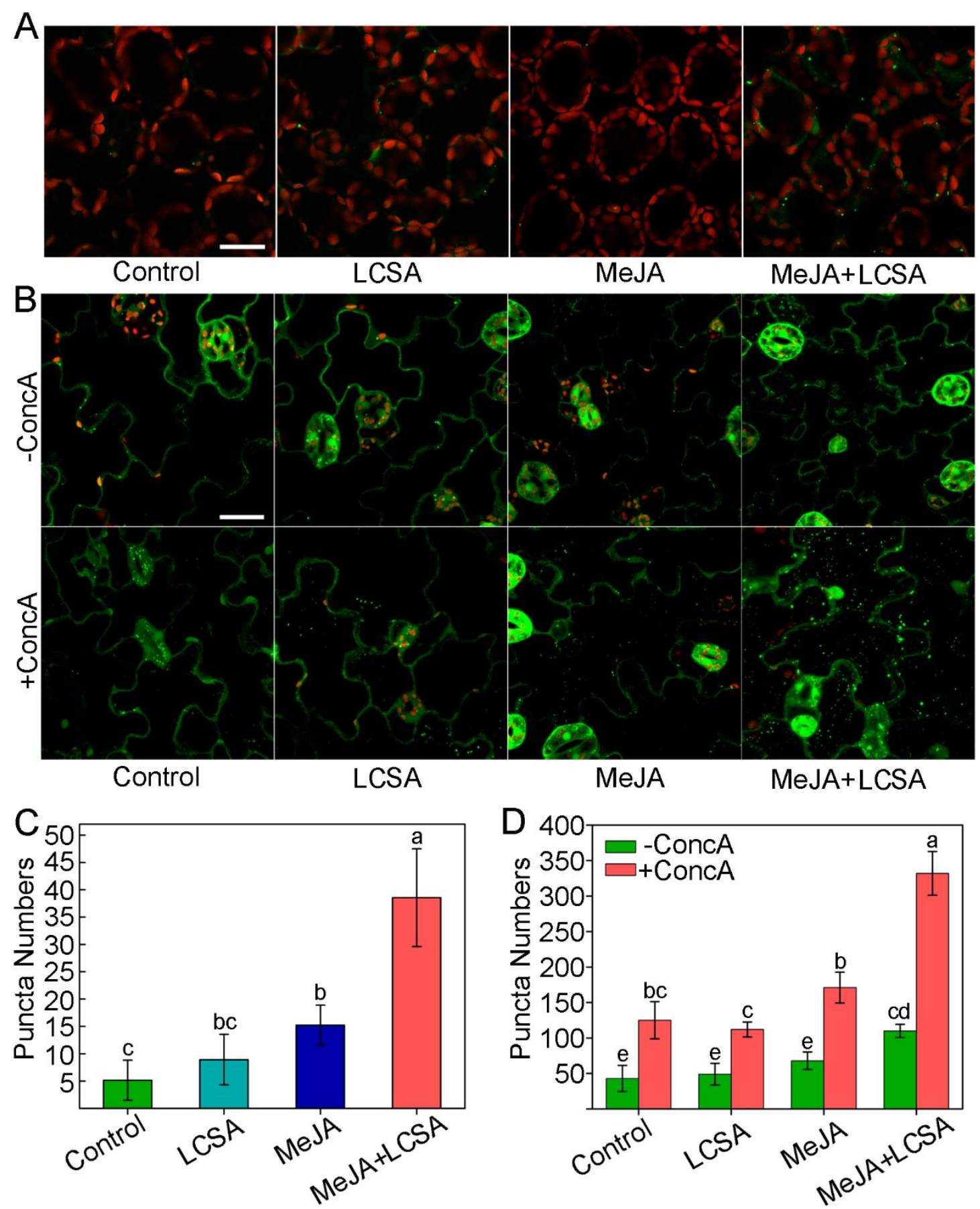

Figure 6. LCSA enhances the formation of autophagosomes upon MeJA-induced leaf senescence. (A) Microscopic analyses of autophagosome-related structures in the mesophyll cells of eYFP-ATG8e plant under LCSA or MeJA or both MeJA and LCSA treatment. (B) Examination of autophagic bodies accumulated in the vacuoles. ConcA, concanamycin A. Bars, $20 \mu \mathrm{m}$. (C and D) Statistical analysis of the puncta numbers displayed in (A) and (B), respectively. The number of puncta was calculated per $0.01 \mathrm{~mm}^{2}$ from at least 15 pictures. This experiment was repeated in triplicate with similar results. Different letters indicate statistically significant differences between the treatments (Duncan's multiple range test, $p<0.05$ ). 
Figures
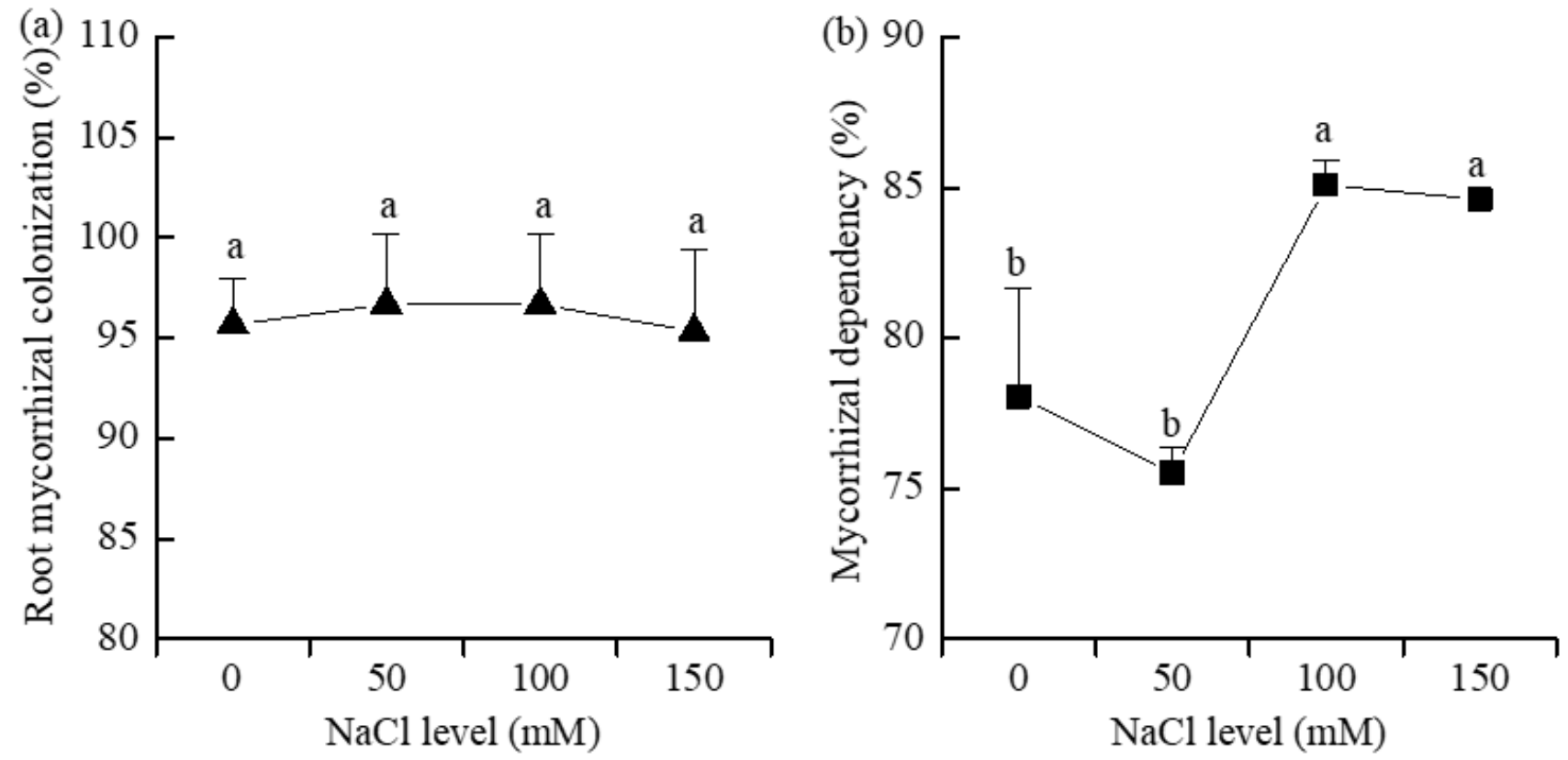

Fig. 1 Mycorrhizal colonization and mycorrhizal dependency of $G$. sinensis seedling at different $\mathrm{NaCl}$ levels. (a) Mycorrhizal colonization, (b) Mycorrhizal dependency. Different lowercase letters indicate significant differences $(P<0.05)$ among $\mathrm{NaCl}$ levels.

\section{Figure 1}

Mycorrhizal colonization and mycorrhizal dependency of G. sinensis seedling at different $\mathrm{NaCl}$ levels. (a) Mycorrhizal colonization, (b) Mycorrhizal dependency. Different lowercase letters indicate significant differences $(P<0.05)$ among $\mathrm{NaCl}$ levels. 


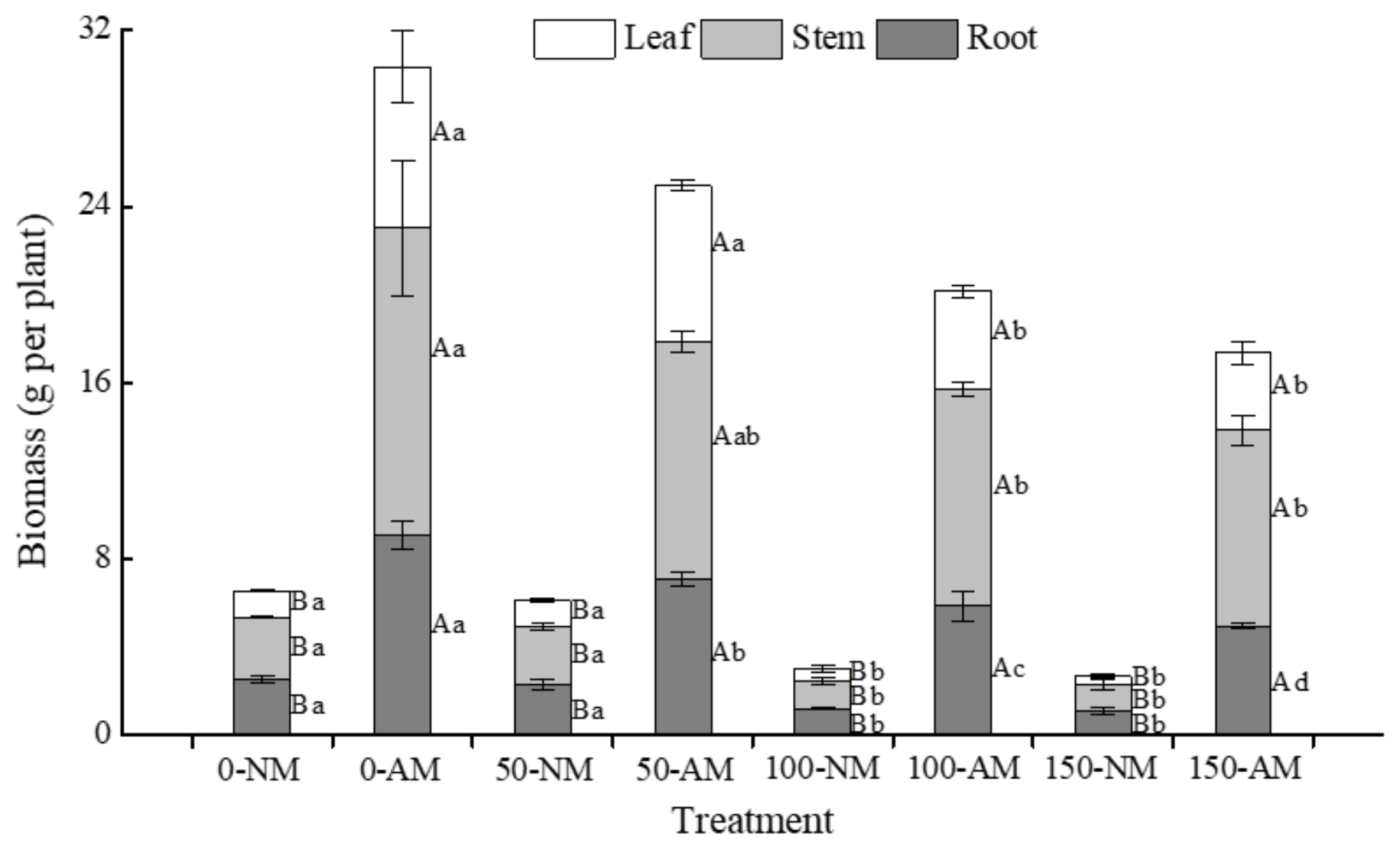

Figure 2

Effects of F. mosseae on biomass of G. sinensis seedling at different $\mathrm{NaCl}$ levels. NM represents the groups without $F$. mosseae inoculation; AM represents the group with $F$. mosseae inoculation. In the same tissues, different capital letters indicate significant differences $(P<0.05)$ among inoculation treatments ( $\mathrm{NM}$ and $\mathrm{AM}$ ) within the same $\mathrm{NaCl}$ level, different lowercase letters indicate significant differences $(\mathrm{P}<$ 0.05 ) among $\mathrm{NaCl}$ levels within the same inoculation treatment. 


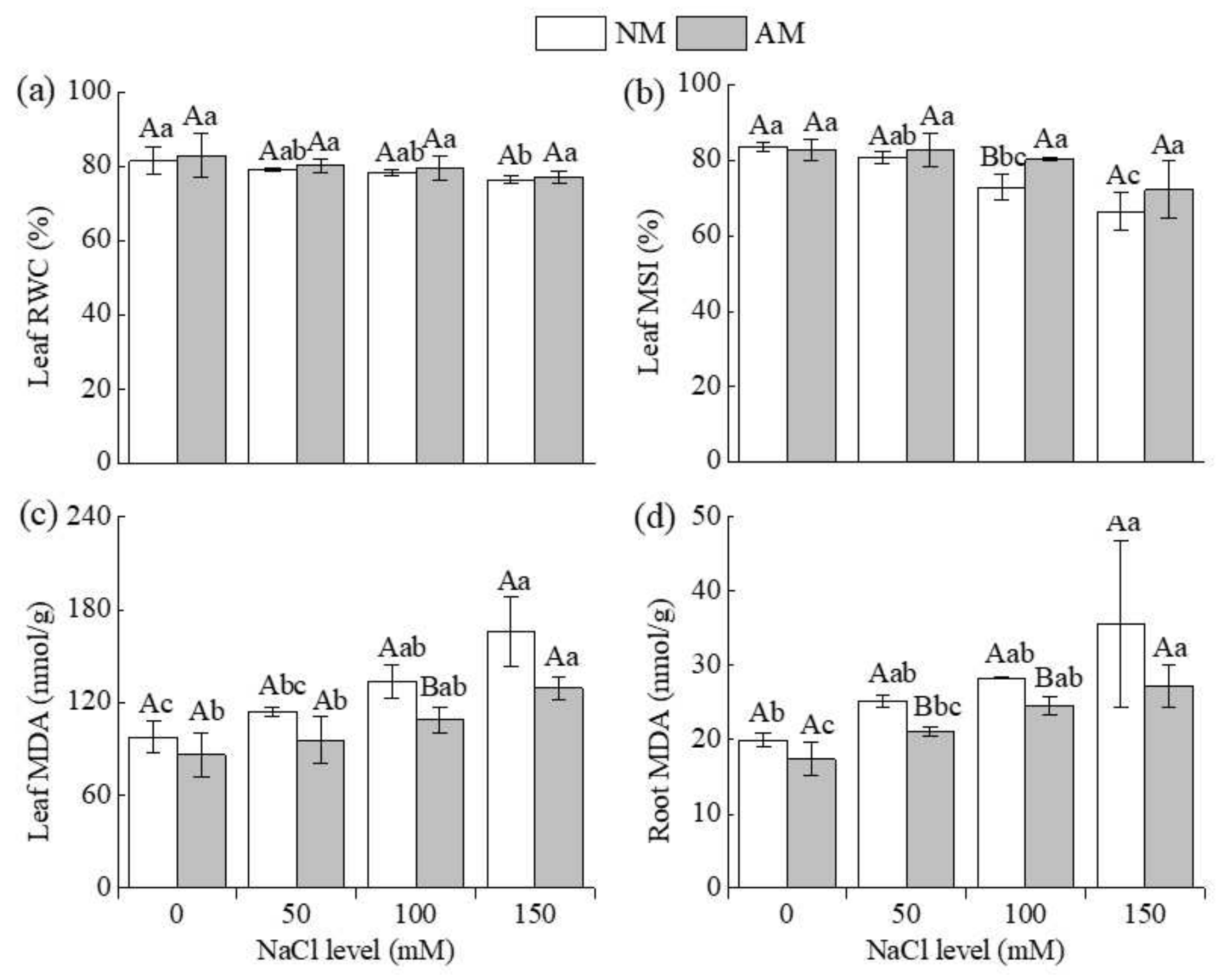

Figure 3

Effects of F. mosseae on MSI, RWC and MDA contents of G. sinensis seedling at different NaCl levels. (a) Leaf relative water content, (b) Leaf membrane stability index, (c, d) Leaf and root MDA; NM represents the groups without $F$. mosseae inoculation; AM represents the group with $F$. mosseae inoculation. Different capital letters indicate significant differences $(P<0.05)$ among inoculation treatments $(N M$ and AM) within the same $\mathrm{NaCl}$ level. Different lowercase letters indicate significant differences $(P<0.05)$ among $\mathrm{NaCl}$ levels within the same inoculation treatment. 

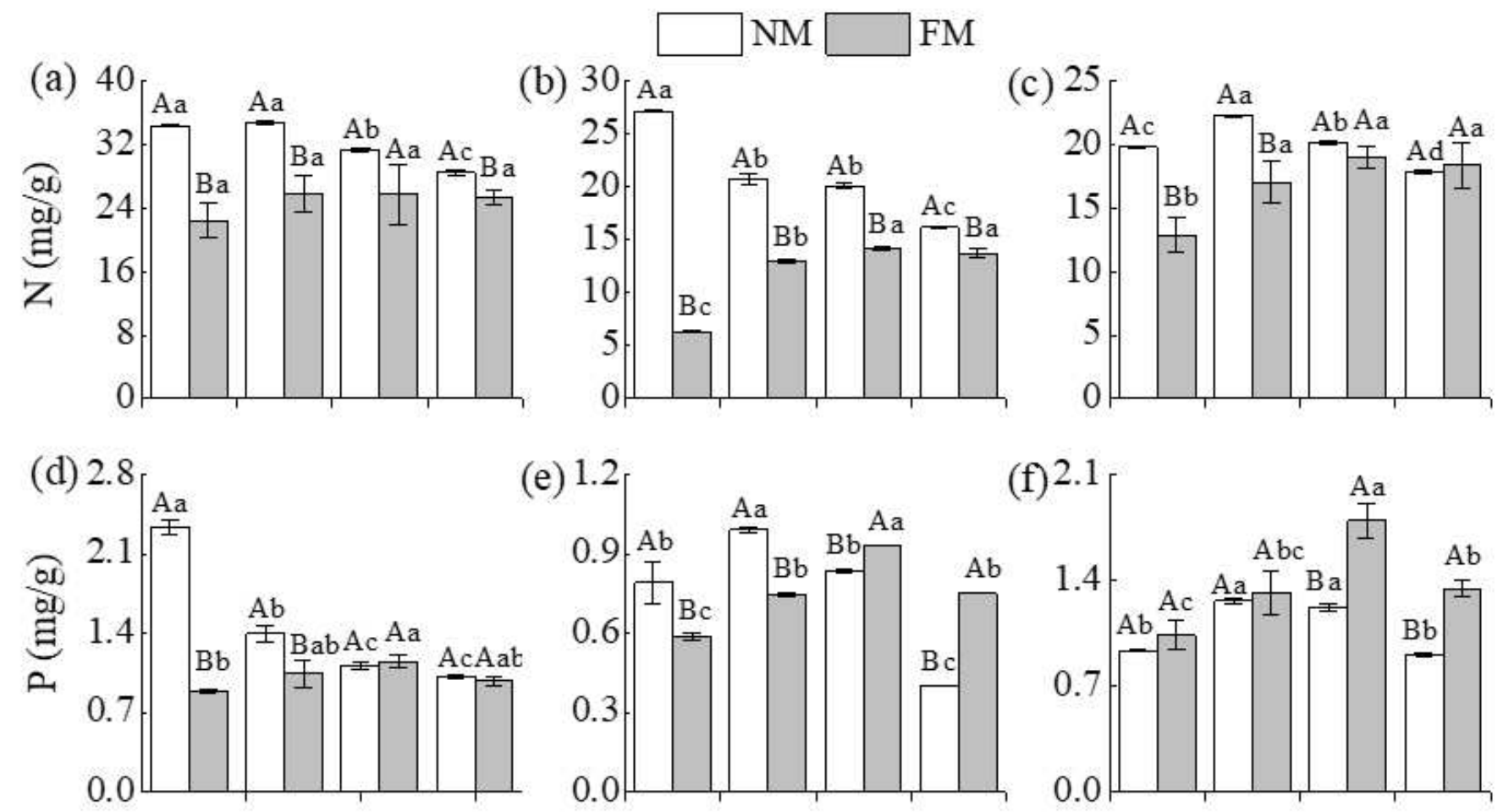

(e) 1.2

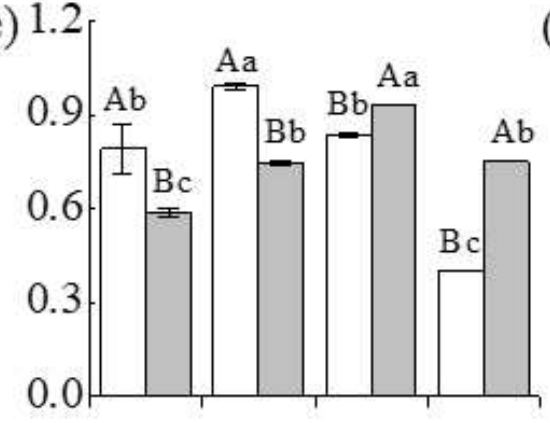

(f) 2.1
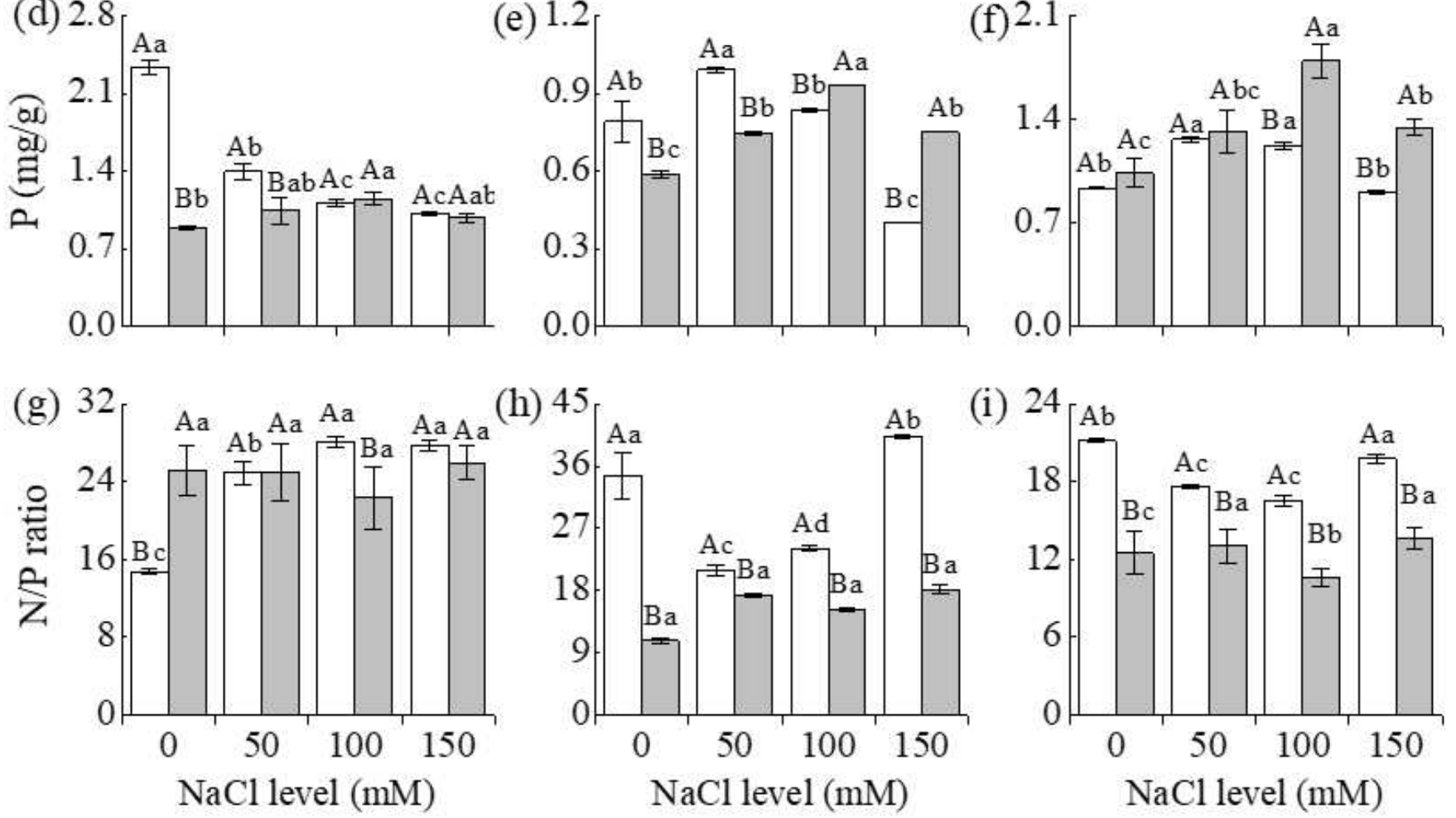

(h) 45

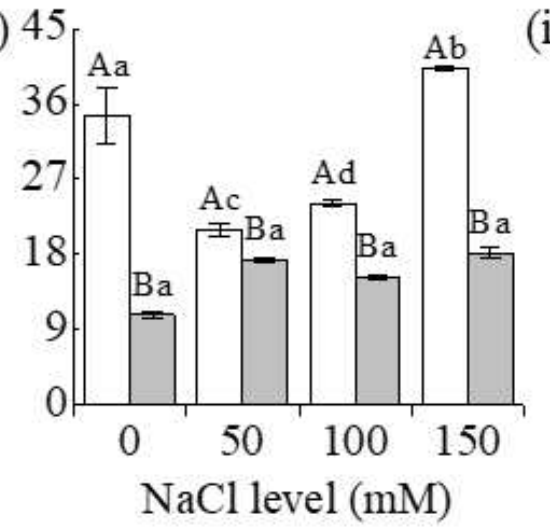

(i)

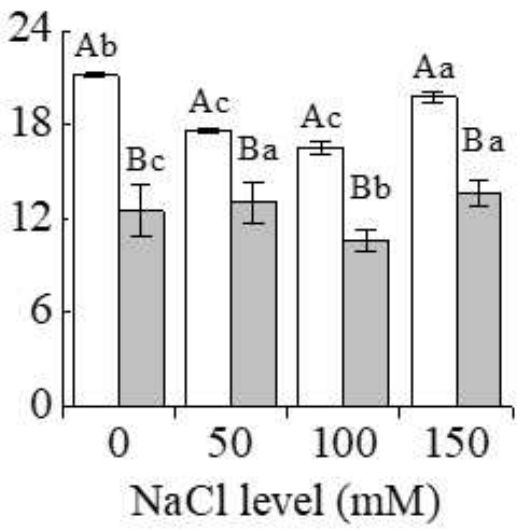

Figure 4

Effects of $\mathrm{F}$. mosseae on N, P concentrations, and N/P ratios of G. sinensis seedling at different $\mathrm{NaCl}$ levels. (a, b c) N concentration in leaf, stem, and root, (d, e, f) P concentration in leaf, stem, and root, (g, h, i) N/P ratio in leaf, stem, and root; NM represents the groups without F. mosseae inoculation; AM represents the group with $\mathrm{F}$. mosseae inoculation. Different capital letters indicate significant differences $(P<0.05)$ among inoculation treatments (NM and $\mathrm{AM})$ within the same $\mathrm{NaCl}$ level. Different lowercase letters indicate significant differences $(P<0.05)$ among $\mathrm{NaCl}$ levels within the same inoculation treatment. 

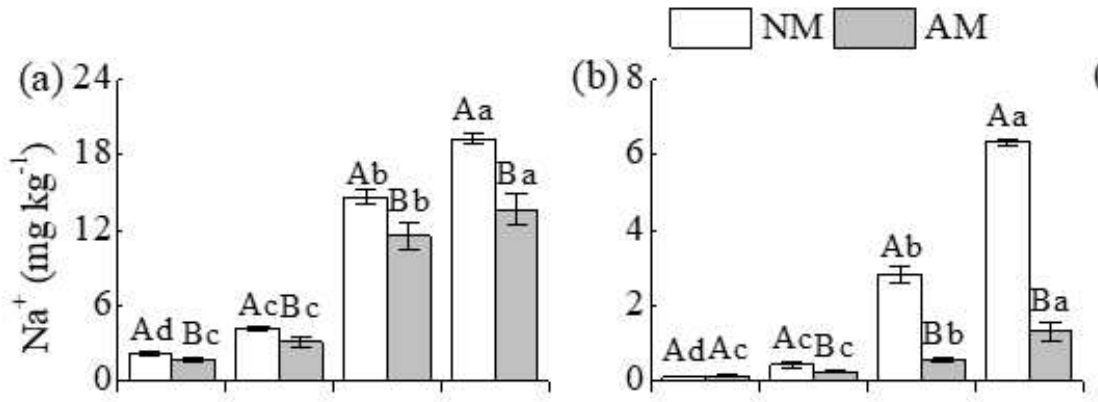

(c) 15
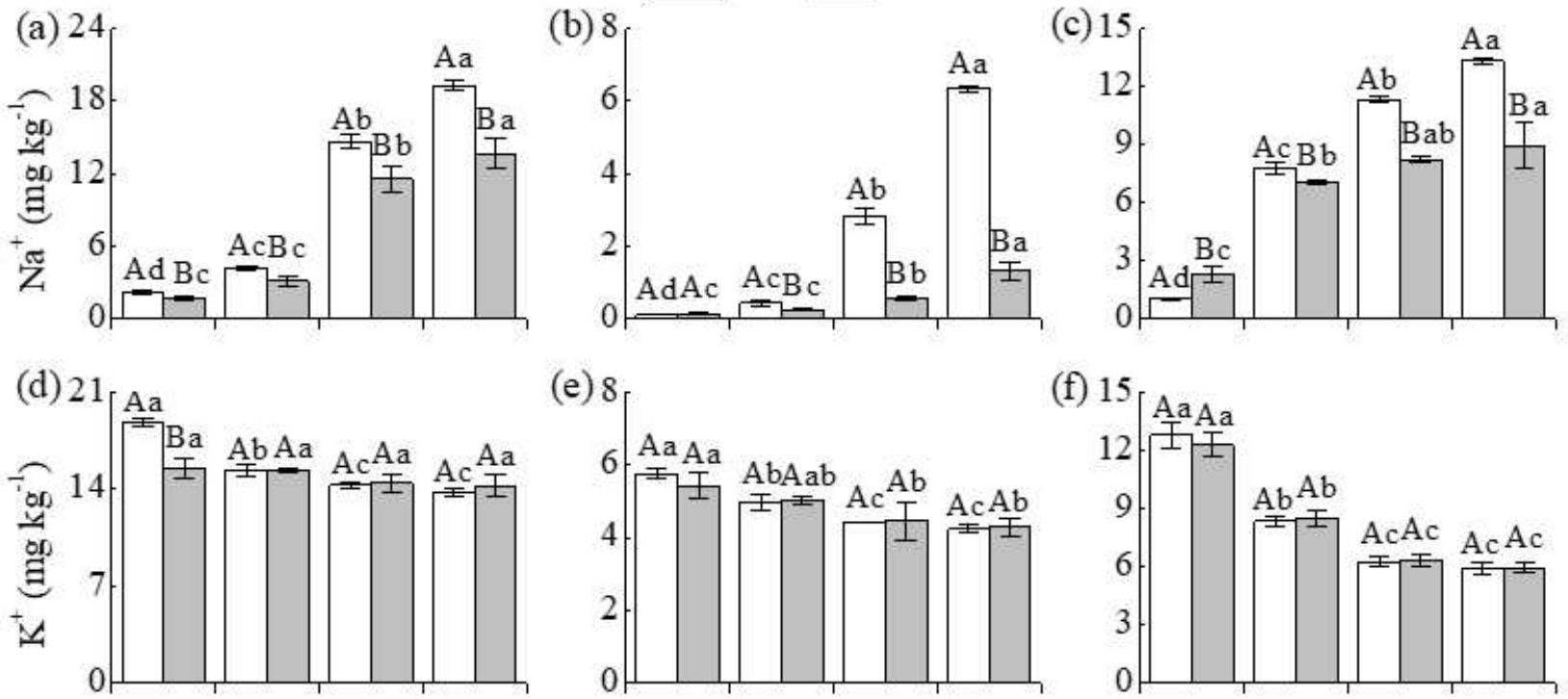

(e) 8

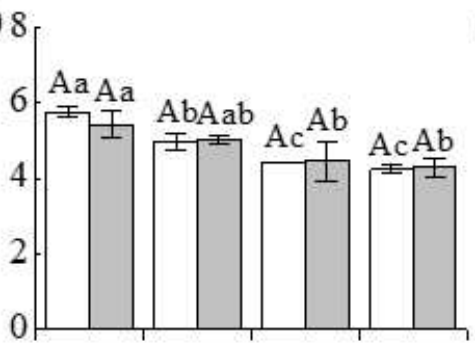

(f) 15
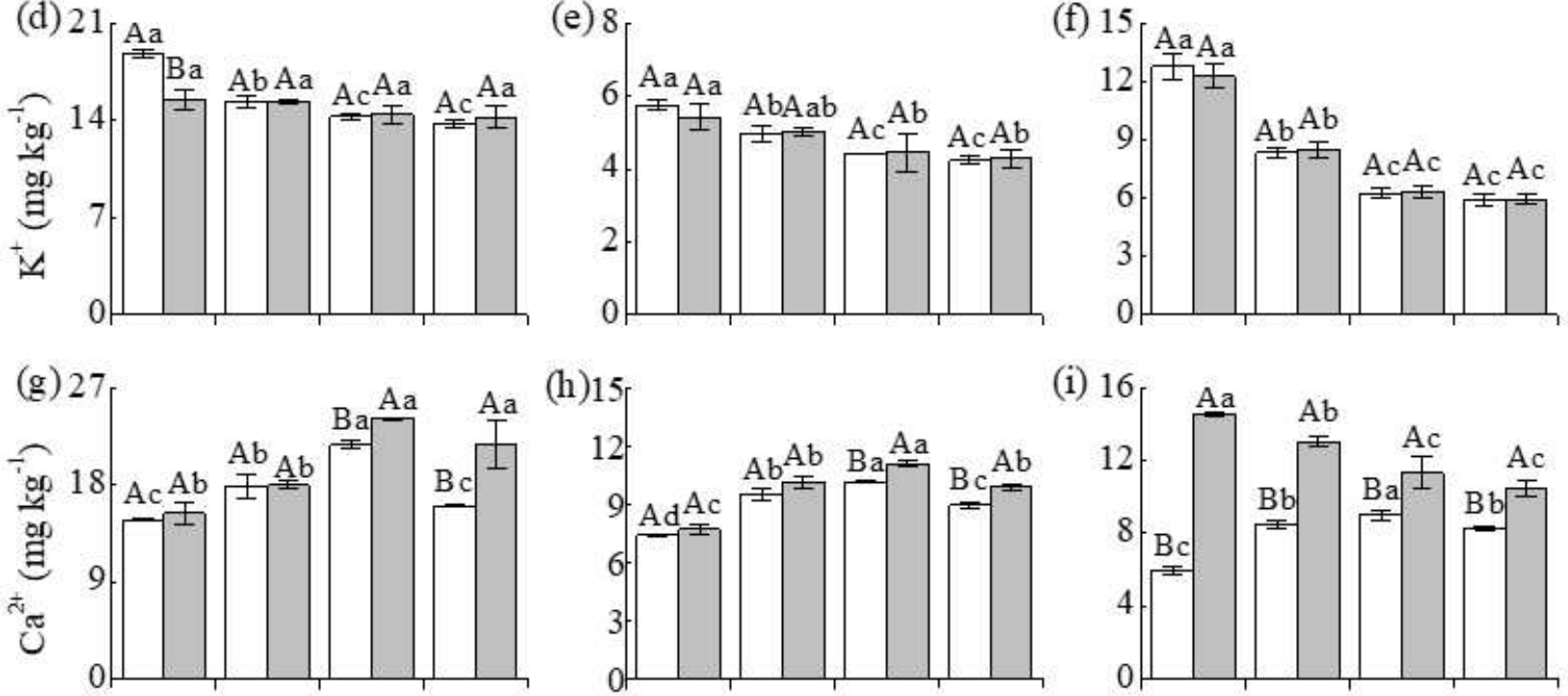

(h) 15

(i) 16
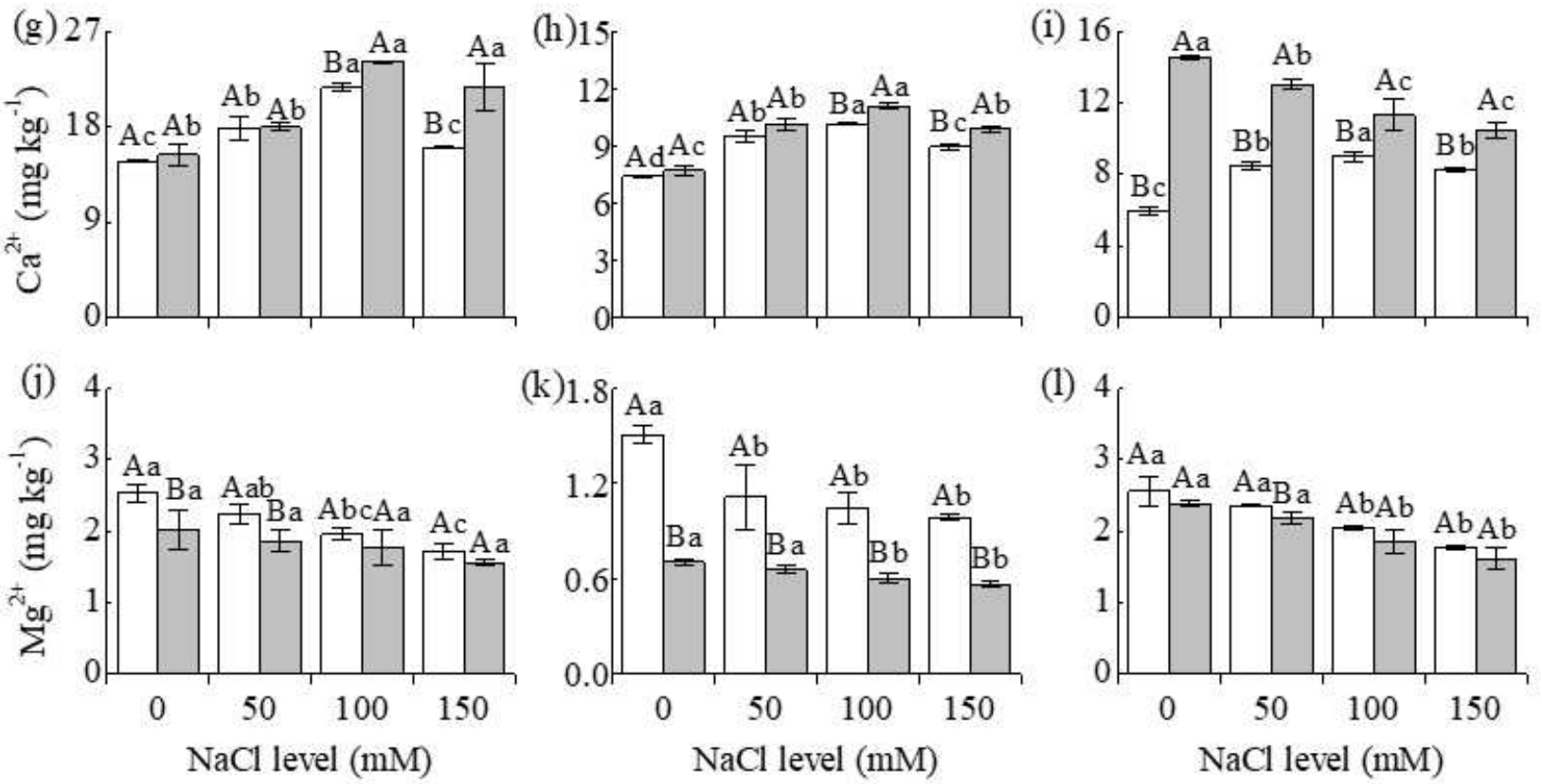

Figure 5

Effects of F. mosseae on the ion concentrations of $\mathrm{G}$. sinensis seedling at different $\mathrm{NaCl}$ levels. $(\mathrm{a}, \mathrm{b}, \mathrm{c})$ $\mathrm{Na}+$ concentration in leaf, stem, and root, (d, e, f) K+ concentration in leaf, stem, and root, (g, h, i) Ca2+ concentration in leaf, stem, and root, (j, k, I) Mg2+ concentration in leaf, stem, and root; NM represents the groups without $F$. mosseae inoculation; AM represents the group with F. mosseae inoculation. Different capital letters indicate significant differences $(P<0.05)$ among inoculation treatments (NM and $A M)$ within the same $\mathrm{NaCl}$ level. Different lowercase letters indicate significant differences $(P<0.05)$ among $\mathrm{NaCl}$ levels within the same inoculation treatment.

\section{Supplementary Files}

This is a list of supplementary files associated with this preprint. Click to download. 
- Supplementarymaterial.docx 